On the spatial development of a dusty wall jet

Duck, P.W. and Hewitt, R.E. and Foster, M.R. 2004

MIMS EPrint: 2012.18

Manchester Institute for Mathematical Sciences

School of Mathematics

The University of Manchester

\footnotetext{
Reports available from: http://eprints.maths.manchester.ac.uk/

And by contacting: The MIMS Secretary

School of Mathematics

The University of Manchester

Manchester, M13 9PL, UK
} 


\title{
On the spatial development of a dusty wall jet
}

\author{
By P. W. DUCK ${ }^{1}$, R. E. HEWITT ${ }^{1}$ AND M. R. FOSTER \\ ${ }^{1}$ Department of Mathematics, University of Manchester, Oxford Road, \\ Manchester, M13 9PL, UK \\ ${ }^{2}$ Department of Aerospace Engineering and Aviation, The Ohio State University, \\ Columbus, OH 43210, USA
}

(Received 5 August 2003 and in revised form 5 May 2004)

We consider the flow of an incompressible particle-laden fluid through the application of the so-called 'dusty-gas' equations, which treat the fluid/particle suspension as two continua. The two phases are described by their individual field equations and interact through a Stokes-drag mechanism. The particular flow we consider is of boundarylayer type, corresponding to the downstream development of a Glauert-type jet adjacent to a horizontal boundary (the inclusion of the particulate phase requires the flow to be non-self-similar). We solve the governing boundary-layer equations through a numerical spatial marching technique in the three distinct cases of (i) weak gravitational influence, (ii) a jet 'above' a wall under the action of gravity and (iii) a jet 'below' a wall under the action of gravity.

The qualitative and quantitative features of the three cases are quite different and are presented in detail. Of particular interest is the development of a stagnation point in the particle velocity field at a critical downstream location in case (i), the development of fluid/particle flow reversal in case (ii) and the development of 'shock' solutions and particle-free regions in case (iii). Asymptotic descriptions are given of the critical phenomena, which support the numerical results. It is found that inclusion of a Saffman force has no substantial effect on either the location or structure of the stagnation-point region.

\section{Introduction}

The interacting flow of an incompressible Newtonian fluid and a dispersed solid particulate phase is a key problem of both fundamental and practical significance. Many flows of environmental and industrial importance involve a fluid within which a distributed, solid, particulate phase exists. Such flows range from waste water ejection in industrial processes, recycling of machine oils, erosion of engineering surfaces by dust and other debris clouds, modification of stability properties in the flows associated with solid propellant devices and a great many others.

These 'dusty gases' have been discussed and examined by a numerous authors over the past 50 years. Important early work was presented by Liu (1966), Saffman (1965), Marble (1963) and Michael (1968), with an early summary provided by Marble (1970). A more recent summary of dusty fluid flows has been given by Osiptsov (1997), in which he thoroughly reviews the development of boundary-layer work since those early papers. No attempt will be made here to repeat his discussion, but there are a few general comments to be made below.

In this paper, we follow a modelling approach that relies on the treatment of the fluid/particulate combination as two interspersed continua. This approach assumes 
that the particles are sufficiently small and of sufficient number to be treated as a continuum, with a well-defined local density and velocity field. These equations may be written down in an ad hoc manner, as in Marble (1970) for example, or formally obtained by a spatial averaging process as in Jackson (1996) and Zhang \& Prosperetti (1997). Closure of the system must involve some hypotheses about the mechanisms through which the two continua interact. Kinetic theory and other models (for examples, see McTigue \& Jenkins 1992; Gundogdu et al. 2003) assume particle interaction through collisions or lubrication forces. A constitutive law for the particle stress tensor, for example, then typically involves a pseudo-temperature, requiring an 'energy' equation to be solved in a coupled way with the other equations. Such models are not appropriate in this very dilute setting, where collisions are rare. Here we shall assume that the particles are sufficiently small, with sufficiently dilute concentration so that inter-phase coupling enters only through a single-particle force law, generally a Stokes-drag term.

There have been some difficulties with various attempts at more general closure schemes, some consequences of which have been discussed by Jones \& Prosperetti (1985) and Prosperetti \& Jones (1985). However, the model equations that we employ here have been used successfully to describe a wide number of flows as in Slater \& Young (2001), Hernández (2001), Narayanan \& Lakehal (2002), Féraille \& Casalis (2003) and Foster, Duck \& Hewitt (2003, referred to hereinafter as FDH).

Particularly relevant to the study at hand are the foundation papers by Singleton (1965), Soo (1968) and Osiptsov (1980), which provide insight into the correct handling of this equation set. FDH used these equations to understand the start-up Kármán problem, discussing some of the subtleties of numerical solutions to these equations, a matter also detailed in Wang \& Glass (1988). As we shall see, these subtleties also come to the fore in the present study.

In summary, the model equations used here are the Navier-Stokes equations for an incompressible Newtonian fluid, coupled via a Stokes-drag interaction to a similar system for the particle phase. However, the particle-'fluid' system lacks any stress tensor. The requirements for the use of these equations has been noted previously, see for example Osiptsov (1997), and are now widely understood to be:

(i) Dilute mono-disperse non-colloidal particle phase-volume fractions of $10 \%$ or less, to avoid particle-particle interactions.

(ii) Heavy particles that make any possible particle pressure negligible, and also imply that added mass effects are negligible.

(iii) Particles that are sufficiently small to permit neglect of the Saffman force; the Stokes force scales with particle radius and the Saffman force with square of the radius. However, later in this work we shall discuss the consequences of including the Saffman force.

Although there is much agreement on the use of the equation set discussed above, there remain some subtleties with regard to the application of boundary conditions on the particle phase. FDH addressed the influence of gravity in the self-similar flow induced by the spin up of a rotating disk. Gravity has a profound influence on the solution structure and on how boundary conditions are to be formulated in a consistent manner. The direction of the gravitational field, relative to the particle drag resulting from the axial boundary-layer transport, is crucial. For example, when the gravitational acceleration is directed towards the rotating boundary, particles are drawn in from the outer extremes of the boundary layer, by both the Ekman suction and gravity, and will ultimately 'collide' with the bounding plane. Under these circumstances, the model does not permit the enforcement of any particle 
boundary conditions on the disk (but rather demands far-field boundary conditions) since the characteristics of the hyperbolic differential operator appearing in the particle phase are directed towards the boundary. Conversely, when the gravitational influence is directed contrary to the local fluid transport, this offers a mechanism whereby particle-free regions can develop with shock-like features in the particle distribution profile. Indeed, similar particle-free flow features have been observed before by other investigators, among them Ungarish \& Greenspan (1983) in rapidly rotating flows. Slater \& Young (2001) also provide a useful discussion of these matters.

These particle-free regions and rapid 'shock'-like transitions in particle concentration are easy to understand from a qualitative perspective. If we have a variable velocity profile in the direction of the local gravitational acceleration, then there is the potential for a balance to exist at a point within the boundary layer where the buoyancy force exerted on a particle balances exactly with the particle-drag force exerted by the fluid motion. Such points may then define boundaries of particlefree regions within the flow and lead to rapid transitions in the particle volume fraction.

The work of FDH addressed the issue of boundary conditions for the particulate phase applied in flow regions that are particle free. This may at first appear to be inappropriate, since we are applying constraints to a phase in a region in which the volume fraction of that phase is zero, however, it was shown in FDH that arbitrary boundary conditions can be imposed in a particle-free region without influencing the solution in regions in which the particle volume fraction is non-zero. This lends support to the approaches taken in numerical modelling of particle-laden flows, see for example Slater \& Young (2001), in which conditions are applied on boundaries irrespective of a zero volume fraction.

In this paper, we seek to extend the ideas developed in FDH to a more general boundary-layer context, making careful use of both numerics and asymptotic methods, as has also been done in Wang \& Glass (1988). The FDH analysis relied upon the application of a self-similar solution to the boundary-layer induced by a rotating disk in a stationary fluid. However, here we shall consider the spatial development of a 'wall-jet' solution, allowing the development of a general spatial form (rather than imposing self-similarity). As we shall see, in the presence of a particle phase with two-way coupling to the fluid, a Glauert similarity solution is no longer appropriate and a more general spatial evolution must be allowed for.

Motivation for this work is centred around the generation of a theoretically self-consistent model of a boundary-layer particle-laden flow, which can then be used to make stringent comparisons with experimental results in a configuration that is feasible to construct in a laboratory. In particular, the lack of interaction between the particulate matter and the boundary, and the importance of additional forces acting on the particles in the presence of a shear flow can potentially be assessed through qualitative/quantitative comparisons and subsequent extensions of the solution method applied here. (We may also note that the deposition characteristics of jet out-falls of particle-laden fluids are a general area of interest in industrial waste water expulsion, although typically a somewhat more complex issue than the problem described here in which both jet and the environment are particle laden.)

We conclude our introduction by noting that, as in the self-similar solution found by Glauert (1956), it is possible to define the flux of the momentum flux. This quantity is invariant in the 'clean' wall jet. If the jet is on the wall $y^{*}=0$ and flowing in the 
$x^{*}$-direction $\dagger$, then this quantity is given by

$$
M \equiv \int_{0}^{\infty} u^{*}\left(y^{*}\right) \int_{y^{*}}^{\infty}\left[u^{*}(s)\right]^{2} \mathrm{~d} s \mathrm{~d} y^{*},
$$

where $u^{*}\left(y^{*}\right)$ is the velocity profile of the jet at/very near the jet source. We introduce this concept here, since the conservation of this quantity (and the analogue in particle-laden flows) will be shown to play a role in the numerical solution and the non-dimensionalization.

The format of this paper is as follows. In $\S 2$, we formulate the problem in the context of the dusty-gas equations, giving details of the numerical scheme and some physical interpretation. In the subsequent sections, we examine three distinct regimes to the problem. In $\S 3$, the zero 'buoyancy' case is investigated, for which gravitational effects are negligible. In $\S 4$, the negative 'buoyancy' case is examined, for which gravitational effects on the particles act towards the plate (which may be regarded as the case of the jet flow above a boundary). In $\S 5$, the regime where gravitational effects act away from the plate is considered (which may be regarded as the case of the jet flow below a boundary). Each of these three regimes exhibits distinct characteristics. In $\S 6$, we provide some discussion on the influence of the Saffman force in our model. Finally, in $\S 7$, our conclusions are presented.

\section{Formulation}

The dimensional equations of motion for the dusty-gas model are conservation of mass,

$$
\nabla^{*} \cdot \boldsymbol{u}^{*}=0, \quad \nabla^{*} \cdot\left(\alpha \boldsymbol{u}_{p}^{*}\right)=0
$$

and the equations of conservation of momentum

$$
\begin{gathered}
\rho_{f}\left(\boldsymbol{u}^{*} \cdot \nabla^{*}\right) \boldsymbol{u}^{*}+\nabla^{*} p^{*}=\mu \nabla^{* 2} \boldsymbol{u}^{*}-\boldsymbol{F}^{*}-\rho_{f} \boldsymbol{g}, \\
\rho_{p} \alpha\left(\boldsymbol{u}_{p}^{*} \cdot \nabla^{*}\right) \boldsymbol{u}_{p}^{*}=\boldsymbol{F}^{*}-\rho_{p} \alpha \boldsymbol{g}+\rho_{f} \alpha \boldsymbol{g} .
\end{gathered}
$$

The quantity $\boldsymbol{u}^{*}$ is the velocity vector of the fluid component, $\boldsymbol{u}_{p}^{*}$ is the velocity vector of the particle phase and $\alpha$ is the particle concentration (assumed small). Note that buoyancy and gravitational terms are present.

Everywhere in the equations where $\alpha$ occurs in an expression $(1-\alpha)$, the $\alpha$ has been neglected. Clearly, when it multiplies other terms (as in the drag term) it must be retained even though small. This is reminiscent of the Boussinesq approximation, but is also asymptotically correct in a formal limit $\alpha \rightarrow 0$ for finite particle loading $\alpha \gamma=O(1)$, where $\gamma \equiv \rho_{p} / \rho_{f}$. The quantities $g$ and $\mu$ are the acceleration due to local gravity and the fluid coefficient of viscosity. The equations seem to be robust in the sense that, when used properly, they give self-consistent answers in a wide variety of problems. We have referenced (in $\S 1$ ) some of this previous work.

Closure of these volume-averaged equations requires that the drag forces per unit volume, $\boldsymbol{F}^{*}$, be specified for the two species. In our case, following a number of previous studies of this type, we shall assume small spherical particles of fixed size,

$\dagger$ Here the asterisk notation ()$^{*}$ indicates dimensional quantities. 
and so we apply the Stokes-drag formula

$$
\boldsymbol{F}^{*}=\frac{9 \mu}{2 a^{2}} \alpha\left(\boldsymbol{u}^{*}-\boldsymbol{u}_{p}^{*}\right)
$$

This formula assumes that $\alpha$ is sufficiently small for the particles to be non-interacting. Wang \& Glass (1988) used both a Stokes-drag term and a different finite-Reynoldsnumber drag law in their Blasius computations, and found no substantial qualitative difference in their results.

We consider a jet flow above the plane $y^{*}=0$, assuming the origin of the jet is located at $x^{*}=0$. Let $U_{\infty}$ be a representative streamwise velocity scale and $L$ be a representative streamwise lengthscale. The streamwise and transverse coordinates are taken to be $L x, L y$, respectively, with the associated fluid $\boldsymbol{u}^{*}=U_{\infty}(U, V)$, and the associated dispersed-phase velocity vector $\boldsymbol{u}_{p}^{*}=U_{\infty}\left(U_{p}, V_{p}\right)$. Crucially, throughout this paper it is assumed that the boundary-layer approximation to (2.1)-(2.3) is appropriate, leading to

$$
\begin{gathered}
\frac{\partial U}{\partial x}+\frac{\partial V}{\partial y}=0, \\
U \frac{\partial U}{\partial x}+V \frac{\partial U}{\partial y}=\frac{\partial^{2} U}{\partial y^{2}}-\alpha \beta\left(U-U_{p}\right), \\
U_{p} \frac{\partial U_{p}}{\partial x}+V_{p} \frac{\partial U_{p}}{\partial y}=\frac{\beta}{\gamma}\left(U-U_{p}\right), \\
U_{p} \frac{\partial V_{p}}{\partial x}+V_{p} \frac{\partial V_{p}}{\partial y}=\frac{\beta}{\gamma}\left(V-V_{p}\right)-\mathscr{K}, \\
\frac{\partial\left(\alpha U_{p}\right)}{\partial x}+\frac{\partial\left(\alpha V_{p}\right)}{\partial y}=0 .
\end{gathered}
$$

The scales $L$ and $U_{\infty}$ are derived later in terms of the flux of the momentum flux and the kinematic viscosity; they are such that $U_{\infty} L=v$.

In this system, three dimensionless parameters appear. Two of these are the fluidparticle density ratio $\gamma$ and a dimensionless measure of the importance of the Stokes-drag force, $\beta \equiv 9 L^{2} / 2 a^{2}$. Here, $a$ is the particle radius, which must be very small compared to the boundary-layer thickness; we return to this matter later in the paper. The third quantity is $\mathscr{K}=g(\gamma-1) L /\left(U_{\infty}^{2} \gamma\right)$ whose magnitude measures the net buoyancy, which can be ignored if this dimensionless number is sufficiently small. (Note that neutral particles make this term zero as well, but the assumptions involved in this model preclude consideration of $\gamma=1$ cases.)

The boundary conditions to be imposed on this system in the case of the fluid are straightforward, namely

$$
\begin{array}{rll}
U=V=0 & \text { on } & y=0, \\
U \rightarrow 0 & \text { as } & y \rightarrow \infty .
\end{array}
$$

The boundary conditions for the particle quantities $\left(\alpha, U_{p}, V_{p}\right)$ are somewhat more subtle (as suggested in the previous section), but in the first instance are taken to be

$$
\alpha \rightarrow \alpha_{\infty}, \quad U_{p} \rightarrow 0 \quad \text { as } \quad y \rightarrow \infty .
$$

One implication of (2.7) is that $\partial V_{p} / \partial y \rightarrow 0$ as $y \rightarrow \infty$. That only one condition is imposed on $U_{p}$ and $\alpha$ (and $V_{p}$ ) is indicative of the first-order nature of the equations 
governing the particle quantities. Certain exceptions to (2.7) will be encountered during the course of the paper, and will be explained as and when appropriate.

The classical ('clean') problem of Glauert (1956) may be retrieved in a variety of ways, most simply by setting $\alpha_{\infty}=0$, and this leads to the similarity structure

$$
(U, V)=\left(x^{-1 / 2} \hat{U}_{0}(\eta), x^{-3 / 4} \hat{V}_{0}(\eta)\right),
$$

where $\eta=y / x^{3 / 4}$, and then $(2.5 a)$ and $(2.5 b)$ yield

$$
\begin{gathered}
-\frac{1}{2} \hat{U}_{0}-\frac{3}{4} \eta \hat{U}_{0 \eta}+\hat{V}_{0 \eta}=0, \\
-\frac{1}{2} \hat{U}_{0}^{2}-\frac{3}{4} \eta \hat{U}_{0} \hat{U}_{0 \eta}+\hat{V}_{0} \hat{U}_{0 \eta}=\hat{U}_{0 \eta \eta},
\end{gathered}
$$

subject to $U_{0}(0)=V_{0}(0)=0, U_{0} \rightarrow 0$ as $\eta \rightarrow 0$. As it stands, this system is effectively homogeneous, but Glauert (1956) showed that the flux of the momentum flux, denoted by $M$ in (1.1), is constant across the jet. $\dagger$ For the purposes of this study, the following normalization procedure (one involving the flux of the momentum flux) was invoked. In these variables, condition (1.1) becomes

$$
\int_{0}^{\infty} \hat{U}_{0}\left(\int_{\eta}^{\infty} \hat{U}_{0}^{2}(\bar{\eta}) \mathrm{d} \bar{\eta}\right) \mathrm{d} \eta=1
$$

provided we require that the velocity and length scales, $U_{\infty}$ and $L$, are related by $M=U_{\infty}^{3} L^{2}$. Although Glauert (1956) showed how an (implicit) solution for $\left(\hat{U}_{0}, \hat{V}_{0}\right)$ is possible, for our purposes, this system was embedded in our overall numerical scheme.

Returning now to the full problem, any singular behaviour to the solution (in particular close to the origin of the jet, $x=0$ ) is probably best treated in the numerical work by properly taking this Glauert structure into account. Asymptotic balancing of terms in (2.5a)-(2.5e) indicates that the following 'caret' variables are appropriate, which should lead to regular behaviour at the origin of the jet, i.e. as $x \rightarrow 0$, whilst at the same time mirror the form of the 'clean' jet (which is expected to be relevant as $x \rightarrow 0$ ). Thus, building in the similarity form suggested by Glauert, whilst allowing the flow to develop spatially downstream, suggests we write

$$
\begin{aligned}
V & =x^{-3 / 4} \hat{V}(\eta, \xi), \\
U & =x^{-1 / 2} \hat{U}(\eta, \xi), \\
V_{p} & =x^{-3 / 4} \hat{V}_{p}(\eta, \xi), \\
U_{p} & =\left(\frac{\beta}{\gamma}\right) x \hat{U}_{p}(\eta, \xi), \\
\alpha & =\hat{\alpha}(\eta, \xi) .
\end{aligned}
$$

The new variable here is $\xi \equiv(\beta / \gamma)^{1 / 2} x^{3 / 4}$, and since there is no independent length scale in this problem (as is the case generally with self-similar flows), if we take the length scale to be the viscous length scale $L=v^{3} / M$, then $U_{\infty}=M / v^{2}$. However, arguments based on other, secondary or artificial length scales, such as the size of the orifice from which the jet emerges, lead ultimately to the same equation set. Therefore,

$\dagger$ Another means is to regard the problem as a (nonlinear) eigenvalue problem for the power of the streamwise decay. 
the non-dimensional coordinates introduced above may be written as follows

$$
x=\frac{M x^{*}}{\nu^{3}}, \quad \xi=x^{3 / 4}\left(\frac{9}{2 \gamma}\right)^{1 / 2}\left(R e_{p}\right)^{-3}, \quad \eta=\left(\frac{M}{v^{3} x^{* 3}}\right)^{1 / 4} y^{*},
$$

where the particle Reynolds number $R e_{p}=(M a)^{1 / 3} / \nu$. Note that since we shall (formally) be assuming that $\xi=O(1)$, then this implies that the streamwise length scale (scaled on the jet thickness) is such that $x \gg 1$, i.e. the streamwise scale is slowly varying compared to the jet thickness. Note that since $x$ may be regarded as the cube of a Reynolds number, it is this requirement that permits the formal use of the boundary-layer approximation. In terms of the caret variables, the system $(2.5 a)-(2.5 e)$ becomes

$$
\begin{gathered}
-\frac{1}{2} \hat{U}-\frac{3}{4} \eta \hat{U}_{\eta}+\frac{3}{4} \xi \hat{U}_{\xi}+\hat{V}_{\eta}=0, \\
-\frac{1}{2} \hat{U}^{2}-\frac{3}{4} \eta \hat{U} \hat{U}_{\eta}+\frac{3}{4} \xi \hat{U} \hat{U}_{\xi}+\hat{V} \hat{U}_{\eta}=\hat{U}_{\eta \eta}-\hat{\alpha} \gamma \xi^{2}\left(\hat{U}-\xi^{2} \hat{U}_{p}\right), \\
\xi^{2} \hat{U}_{p}^{2}-\frac{3}{4} \xi^{2} \eta \hat{U}_{p} \hat{U}_{p \eta}+\frac{3}{4} \xi^{3} \hat{U}_{p} \hat{U}_{p \xi}+\hat{V}_{p} \tilde{U}_{p \eta}=\hat{U}-\xi^{2} \hat{U}_{p}, \\
\hat{V}_{p} \hat{V}_{p \eta}+\frac{3}{4} \xi^{2} \hat{U}_{p}\left[-\hat{V}_{p}-\eta \hat{V}_{p \eta}+\xi \hat{V}_{p \xi}\right]=\xi^{2}\left[\hat{V}_{-}-\hat{V}_{p}\right]-\mathscr{K}(\gamma / \beta)^{3 / 2} \xi^{3}, \\
\hat{V}_{p} \hat{\alpha}_{\eta}+\hat{\alpha} \hat{V}_{p \eta}+\xi^{2} \hat{\alpha}\left[\hat{U}_{p}-\frac{3}{4} \eta \hat{U}_{p \eta}+\frac{3}{4} \xi \hat{U}_{p \xi}\right]+\frac{3}{4} \hat{U}_{p}\left[-\eta \hat{\alpha}_{\eta}+\xi \hat{\alpha}_{\xi}\right] \xi^{2}=0 .
\end{gathered}
$$

Thus, the quantity $\beta$, which under the required choice of $L$ is now given by $9 \operatorname{Re}_{p}^{-6} / 2$, is entirely scaled out of the problem apart from its presence in the buoyancy term, which is now given by

$$
\mathscr{K}^{\prime} \equiv\left(\frac{\gamma}{\beta}\right)^{3 / 2} \mathscr{K}=\frac{g a^{3}}{v^{2}}\left(\frac{2 \gamma}{9}\right)^{3 / 2}\left(1-\frac{1}{\gamma}\right) .
$$

This quantity is often called a 'Galileo number' in the particle-flow literature. Note, too, that the volume fraction occurs in the combination $\alpha \gamma$, that is, the "particle loading'.

The 'dusty' counterpart to (2.10) is the following integral condition

$$
\int_{0}^{\infty} \hat{U} \int_{\eta}^{\infty} \hat{U}^{2} \mathrm{~d} \bar{\eta} \mathrm{d} \eta-\int_{0}^{\xi} \int_{0}^{\infty} \xi \hat{U} \int_{\eta}^{\infty} \gamma \hat{\alpha}\left(\hat{U}-\xi^{2} \hat{U}_{p}\right) \mathrm{d} \bar{\eta} \mathrm{d} \eta \mathrm{d} \xi=1 .
$$

Near the source of the jet (that is, as $\xi \rightarrow 0$ ), we recover the Glauert similarity equations for the fluid (2.9) and the corrections to the fluid velocity field due to the influence of the suspended particle phase are $O\left(\xi^{2}\right)$. In this regard, from a numerical point of view, it is convenient to seek a form of solution that (even) more precisely builds in the correct near-origin behaviour of the singular jet, namely,

$$
\begin{aligned}
\hat{V}(\eta, \xi) & =\hat{V}_{0}(\eta)+\xi^{2} \tilde{V}(\eta, \xi), \\
\hat{U}(\eta, \xi) & =\hat{U}_{0}(\eta)+\xi^{2} \tilde{U}(\eta, \xi), \\
\hat{V}_{p}(\eta, \xi) & =\hat{V}_{0}(\infty)-\mathscr{K}^{\prime} \xi+\xi^{2} \tilde{V}_{p}(\eta, \xi), \\
\hat{U}_{p}(\eta, \xi) & =\tilde{U}_{p}(\eta, \xi), \\
\hat{\alpha}(\eta, \xi) & =\alpha_{\infty}+\xi^{2} \tilde{\alpha}(\eta, \xi) .
\end{aligned}
$$

Substitution of (2.16) into (2.13) yields alternative governing equations; it is these equations that are employed in our numerical work. 
The key points to note in the above formulation are that: (i) as $\xi \rightarrow 0$, all caret and tilde quantities are regular and bounded (indeed, these limiting values were generally used as initial conditions in our calculations); (ii) as $\eta \rightarrow \infty, \tilde{U}, \hat{U}, \tilde{U}_{p}, \hat{U}_{p} \rightarrow 0, \hat{\alpha} \rightarrow \alpha_{\infty}$ (implying $\tilde{\alpha} \rightarrow 0$ ), $\hat{V}_{p} \rightarrow \hat{V}-\mathscr{K}^{\prime} \xi$ (implying $\tilde{V}_{p} \rightarrow \tilde{V}$ ); (iii) at the origin of the jet, the normal particle velocity (to leading order) is just that of the outer-edge transpiration velocity of the fluid $\left(\hat{V}_{0}(\infty) \approx-0.628<0\right)$, and the next order, correction term to this is just the buoyancy influenced component, $-\mathscr{K}^{\prime} \xi$; the comments in the previous section regarding the potential balance between transpiration and buoyancy effects are likely, therefore, to be an issue if $\mathscr{K}^{\prime}<0$.

Some calculations were performed in which the flow was treated as 'clean' up to $\xi=1$, at which location distributions of $\hat{U}_{p}(\eta, \xi=1), \hat{V}_{p}(\eta, \xi=1), \hat{\alpha}(\eta, \xi=1)$ were imposed on this flow; the motivation in this case was to mimic the injection of particles at a finite downstream location. A calculation of this type is presented in Appendix.

\section{Parametric restrictions}

Before proceeding, we note that the requirement that the particle size be small relative to the boundary-layer thickness

$$
a \ll\left(\frac{v^{3} x^{* 3}}{M}\right)^{1 / 4},
$$

can be written as

$$
\frac{M x^{*}}{v^{3}} \gg R e_{p}^{4}
$$

Since $R e_{p}$ must be small to guarantee validity of the Stokes-drag formula, this restriction is less severe than the requirement for the validity of boundary-layer theory itself, namely, $M x^{*} / v^{3} \gg 1$ (the latter merely presupposing that the streamwise scale is considerably larger than the boundary-layer thickness).

\subsection{The Saffman force}

Some investigators, for example Lee \& Chan (1972) and Asmolov (1992), include a 'Saffman force' (Saffman 1965) in the governing equations. Though such a force does not typically result from the formal processes employed by Jackson (1996) or Zhang \& Prosperetti (1997), H. H. Shen (personal communication, 2003) argues that this is because the force summation on the dispersed phase is simply set equal to the Stokes drag, rather than a vector sum of the Stokes drag and the Saffman force. Incorporating this modifies only equation $(2.13 d)$, adding to the right-hand side of this equation the expression

$$
c\left(\frac{2}{9}\right)^{3 / 4} \gamma^{-1 / 4} \xi^{3 / 2}\left[\hat{U}-\xi^{2} \hat{U}_{p}\right]\left|\hat{U}_{\eta}\right|^{1 / 2} \operatorname{Re}_{p}^{3 / 2} s,
$$

where $c$ is a numerical constant, and $s$ is a sign adjustment depending on the flow direction. Note that the assumed smallness of $R e_{p}$ makes this term always smaller than the Stokes-drag term, with potential importance arising only if the gradient of the fluid velocity becomes very large. We return to this question later, to enquire into whether or not this force alters the non-uniformities that arise at a finite location downstream. 


\section{The numerical marching procedure}

The governing equations together with the integral constraint (2.15) then form the basis of our numerical investigation, which involves a standard (Crank-Nicolson) marching procedure in $\xi$ (together with second-order differencing in $\eta$ ); consequently, the scheme is overall of second-order accuracy. Poor quality treatment of the governing equations near $\xi=0$ invariably leads to spurious growing oscillations in the computed solution downstream. Therefore, in developing the numerical results presented herein, great care has been taken to ensure numerical accuracy. In particular, the use of the tilde variables in (2.16) (together with their appropriate limiting values as $\xi \rightarrow 0$ ) is crucial in this respect. Further, any artificial parameters associated with the numerical scheme, such as the grid size across the boundary layer, the spatial step size in the streamwise marching process and the position at which the 'far field' conditions are applied, have no influence on the results (as graphically presented) when refined further. Systematic studies of the convergence of the solutions with respect to the domain size and spatial resolution were performed for a range of physical parameters, but are not presented here. Typical grid parameters chosen consist of a transverse grid of $\Delta \eta=0.02$ (extending out to $\eta=20$ ), and a streamwise stepsize of $\Delta \xi=2.5 \times 10^{-4}$. All flow quantities at a given streamwise location were simultaneously calculated (using Newton iteration), with the banded structure of the resulting algebraic system being fully exploited. Typical computation times were a few minutes on a $2.5 \mathrm{GHz}$ Pentium 4 processor.

The results obtained in this way are described in the subsequent sections. As usual, if either of $\hat{U}$ or $\hat{U}_{p}$ changes sign, corresponding to a change of streamwise direction of the fluid or the particles, respectively, then the nature of the governing equations profoundly changes and the concept of streamwise marching is no longer appropriate.

Finally, although the main focus of our attention in this paper is on the 'full' system $(2.13 a)-(2.13 e)$, later in the paper it will be useful to refer to a dilute limit - this refers to the limit $\alpha \rightarrow 0$, i.e. vanishingly small particle concentration levels so that the fluid affects the particle motion, but the particles do not affect the fluid motion. We must note that this is distinct from the 'flow visualization' limit, which is formerly achieved for vanishingly small particle size, that is, $\beta \rightarrow \infty$. Quite simply, the 'dilute limit' is achieved by setting $\hat{\alpha}=0$ in (2.13a), which in turn implies (generally) that $\tilde{U}=\tilde{V}=0$, and so the fluid motion is merely that corresponding to a particle-free jet. As a consequence, the similarity form (for the fluid motion) is maintained throughout the streamwise extent of the flow, although the corresponding problem for the particle motion (obtained by solving $(2.13 c)-(2.13 e))$ is non-self-similar.

\subsection{The physical interpretation}

The flow we are describing here is the spatial development of a two-dimensional jet in an incompressible fluid issuing near a wall at some prescribed location $x=0$. If we ignore the role of the suspended particles for a moment, then the behaviour is the classical Glauert similarity solution, an exact reduction of the Navier-Stokes equations. For a flow that evolves according to the Glauert self-similarity, we find a jet that broadens as it evolves along the bounding wall (thickening like $x^{3 / 4}$ ) and algebraically decaying with $x^{-1 / 2}$ and $x^{-3 / 4}$ factors for the streamwise and transverse velocity components, respectively. We must note that the inclusion of a particle phase suspension removes any possibility of a self-similar flow (this is immediately apparent by inspection of $(2.13 a)-(2.13 e))$. 
(a)

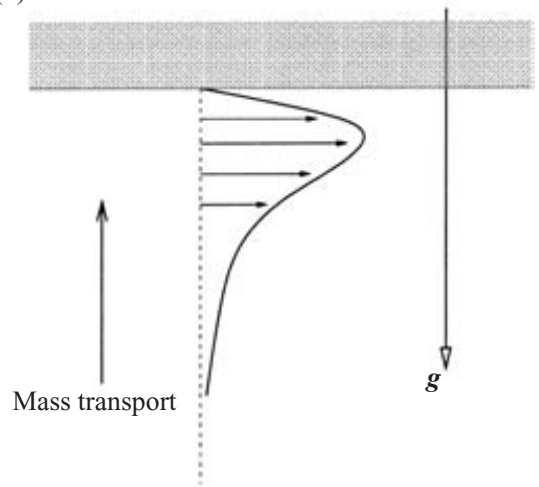

(b)

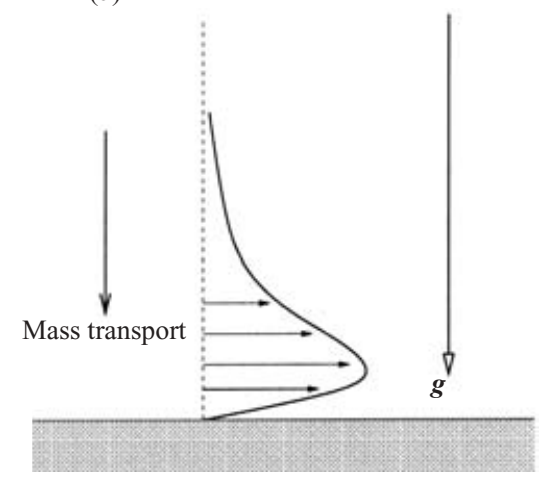

FIgURE 1. The two cases of $(a) \mathscr{K}^{\prime}<0$ (local gravitational acceleration is directed in the opposite sense as the far-field fluid transport induced by the layer) and $(b) \mathscr{K}^{\prime}>0$ (local gravitational forcing is in the same direction to the far-field mass transport induced by the boundary layer). The case $\mathscr{K}^{\prime} \ll 1$ corresponds to problems in which the gravitational influence is weak.

It is key to the interpretation of the results presented in this work that, as the 'Glauert'-type wall jet develops, there is a spatially decaying mass flux into the boundary layer from the external flow; this is induced by the lateral transport inside the layer. Near the singular source of the jet, this transpiration is dominant and the Stokes-drag forcing induced in the particle phase results in a concomitant net transport in the suspension in the same direction, that is, the particles are dragged by the fluid towards the wall.

We shall consider three cases in this paper, namely $\mathscr{K}^{\prime}=0, \mathscr{K}^{\prime}>0$ and $\mathscr{K}^{\prime}<0$ (in $\S \S 3,4$ and 5 , respectively). These are shown schematically in figure 1 , with the two different signs of $\mathscr{K}^{\prime}$ corresponding to the wall jet being above or below the boundary (we assume throughout this work that the particles are significantly heavier than the surrounding fluid in which they are embedded).

We can now see why one might anticipate these cases to be qualitatively different, since for $\mathscr{K}^{\prime}>0$ both the Stokes-drag forcing of the particles (due to the net vertical fluid transport induced in the wall jet) and the gravitational forcing act towards the wall. However, for $\mathscr{K}^{\prime}<0$, these two forcing mechanisms compete, with the transpiration induced by the boundary layer lifting particles towards the wall, whilst gravity acts to oppose this motion.

For $\mathscr{K}^{\prime}<0$, near the origin the singular nature of the wall jet is the dominant mechanism, transporting particles in the opposing direction to the local gravitational acceleration. Nevertheless, the streamwise decay of the wall jet requires that the mass transport into the boundary layer must decrease, eventually leading to a point at which the interplay of the Stokes drag and gravitational forcing can become more subtle. The competition between these two forcing mechanisms is addressed in some detail in this paper.

In terms of the mathematical viewpoint, the hyperbolic nature of the equations governing the particle phase means that a change in the sign of $\hat{V}_{p}$ leads to a change in the direction of the characteristics in the $(\eta, \xi)$-plane (here $\xi$, the streamwise coordinate, plays a time-like role). The direction of the characteristics is crucial to the choice of boundary conditions on the particle phase and also the potential 


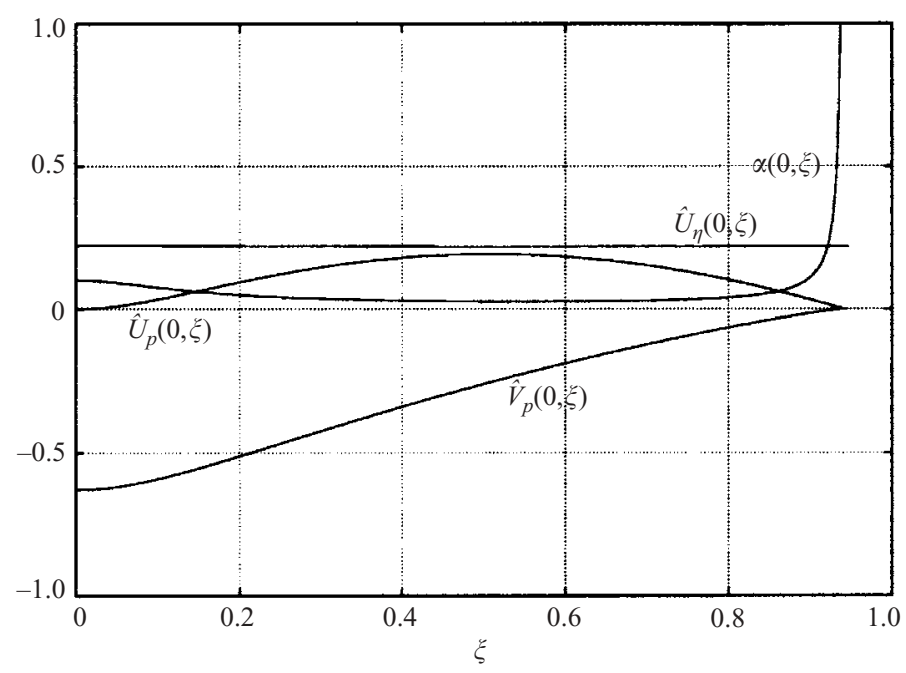

FIGURE 2. Downstream development of surface quantities, $\mathscr{K}^{\prime}=0, \alpha_{\infty} \gamma=0.1$.

development of particle-free regions in the flow (or equivalently, regions of high particle concentration).

\section{The case $\left|\mathscr{K}^{\prime}\right| \ll 1$ : weak gravitational forcing}

We first consider flows for which $\left|\mathscr{K}^{\prime}\right| \ll 1$, that is gravitational effects are negligible. Figure 2 shows the downstream flow and particle development for the case $\alpha_{\infty} \gamma=0.1$ (note that the structure of the flow in $(\eta, \xi)$ space is dependent on a single parameter $\left.\mathscr{K}^{\prime}\right)$. Shown are the four key surface quantities, namely the wall shear stress of the fluid $\hat{U}_{\eta}(0, \xi)$, the particle slip ( $\xi$-wise) velocity at the wall $\hat{U}_{p}(0, \xi)$, the particle normal ( $\eta$-wise) velocity component at the wall $\hat{V}_{p}(0, \xi)$, and the particle concentration on the surface $\alpha(0, \xi)$. In this case, the response of the fluid is relatively benign, with $\hat{U}_{\eta}(0, \xi)$ varying only slightly on the scale shown, however, there is a significant response observed in the particle quantities. At a critical streamwise location (denoted by $\xi_{s}$, in this case $\approx 0.94$ ), the particle concentration increases significantly and it was not possible to continue calculations beyond this point. It should be emphasized that this violates one of our initial assumptions, namely that $\alpha \ll 1$, but nonetheless, given the extremely rapid build-up in the surface value of $\alpha$, we may surmise there is indeed some physical significance to this observation. Furthermore, all the indications are that the two components of the particle surface velocity, $\hat{U}_{p}(0, \xi)$ and $\hat{V}_{p}(0, \xi)$ both become zero simultaneously at this critical event (see figure 3). A number of very careful numerical experiments were performed, on a variety of grids, and these all confirmed this assertion. Physically, the simultaneous development of (effectively) a stagnation point in the particle flow with the development of a build up of fluid particles (described by a rapid growth in particle concentration) seems entirely plausible. We will consider this important point next.

Note that the actual distance in terms of $x$ is dependent on the actual value of $R e_{p}$, but in these coordinates, the location is universal. The results detailed above, together with other numerical results performed by the authors, and dominant asymptotic balancing of terms, point to the following flow development as $\xi \rightarrow \xi_{s}$ 


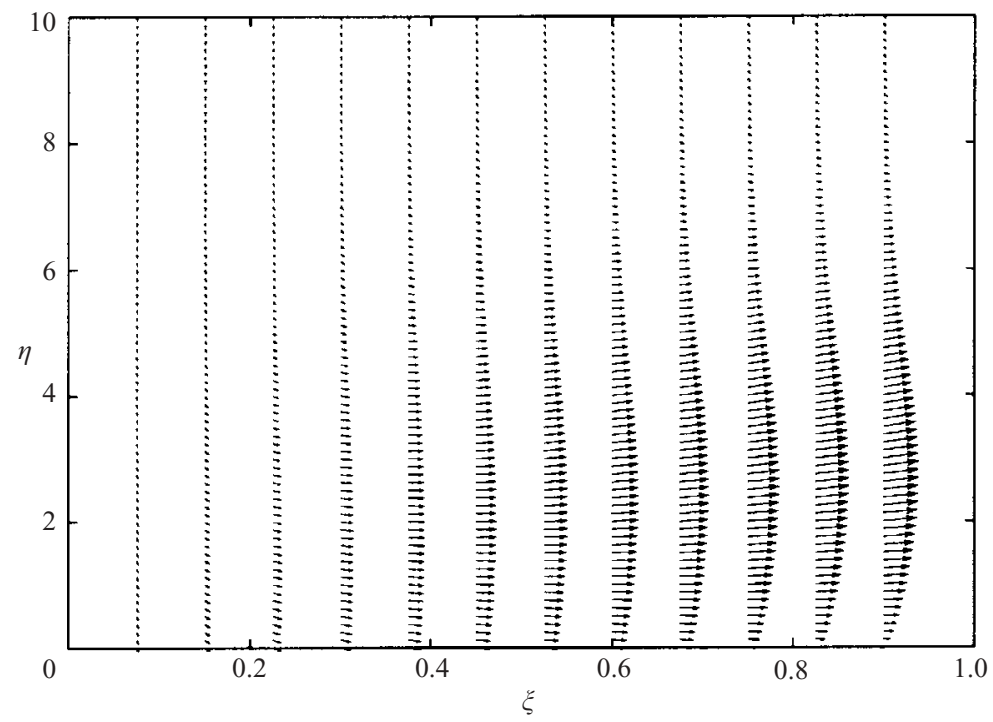

FIGURE 3. Downstream development of particle velocity vector, $\mathscr{K}^{\prime}=0, \alpha_{\infty} \gamma=0.1$.

near the wall:

$$
\begin{aligned}
\hat{U}_{p} & =\frac{4}{3} \frac{\left(\xi_{s}-\xi\right)}{\xi_{s}} \hat{U}_{p 0}(\hat{\eta})+\cdots, \\
\hat{V}_{p} & =\frac{4}{3}\left(\xi_{s}-\xi\right) \xi_{s}^{3} \hat{V}_{p 0}(\hat{\eta})+\cdots, \\
\alpha & =\left(\xi_{s}-\xi\right)^{-1} \alpha_{0}(\hat{\eta})+\cdots,
\end{aligned}
$$

where $\hat{\eta}=\frac{3}{4} \eta /\left[\left(\xi_{s}-\xi\right) \xi_{s}\right]=O(1)$.

It is our conjecture that at/near this point, the fluid behaviour is benign, and the streamwise component of the flow, in the $\hat{\eta}=O(1)$ region can be represented merely by

$$
\left(\frac{4}{3} \xi_{s}\left(\xi_{s}-\xi\right) \hat{U}_{\eta}\left(0, \xi_{s}\right) \hat{\eta}, \quad \frac{8}{9} \xi_{s}^{2}\left(\xi_{s}-\xi\right)^{2} \hat{V}_{\eta \eta}\left(0, \xi_{s}\right) \hat{\eta}^{2}\right) .
$$

Substitution of $(3.1 a)-(3.1 c)$ into $(2.13 c)-(2.13 e)$ leads to the system

$$
\begin{gathered}
-\hat{U}_{p 0}^{2}+\hat{\eta} \hat{U}_{p 0} \hat{U}_{p 0 \hat{\eta}}+\hat{V}_{p 0} \hat{U}_{p 0 \hat{\eta}}=\hat{U}_{\eta}\left(\eta=0, \xi_{s}\right) \hat{\eta}-\hat{U}_{p 0}, \\
-\hat{V}_{p 0} \hat{U}_{p 0}+\hat{\eta} \hat{U}_{p 0} \hat{V}_{p 0 \hat{\eta}}+\hat{V}_{p 0} \hat{V}_{p 0 \hat{\eta}}=-\hat{V}_{p 0}, \\
\hat{\eta} \hat{U}_{p 0 \hat{\eta}} \alpha_{0}+\hat{\eta} \hat{\alpha}_{0 \hat{\eta}} \hat{U}_{p 0}+\hat{V}_{p 0} \alpha_{0 \hat{\eta}}+\hat{V}_{p 0 \hat{\eta}} \alpha_{0}=0 .
\end{gathered}
$$

In order to match with the flow above this sublayer (i.e. in the $\eta=O(1)$ zone, wherein all quantities are $O(1)$ themselves), we require as $\hat{\eta} \rightarrow \infty$ that $\hat{U}_{p 0}, \hat{V}_{p 0}=O(\hat{\eta})$, whilst $\alpha_{0}=O\left(\hat{\eta}^{-1}\right)$. Consequently, it appears that the critical value $\xi_{s}$ is a function of $\alpha_{\infty} \gamma$, but the structure near $\xi=\xi_{s}$ is universal for $\mathscr{K}^{\prime} \equiv 0$.

\section{The case $\mathscr{K}^{\prime}>0$ : flow above a boundary}

In this case, gravitational effects act in the same (downwards) direction as the initial jet entrainment $\left(\right.$ remembering $\hat{V}_{0}(\infty)<0$ ). Figure 4 shows the downstream 


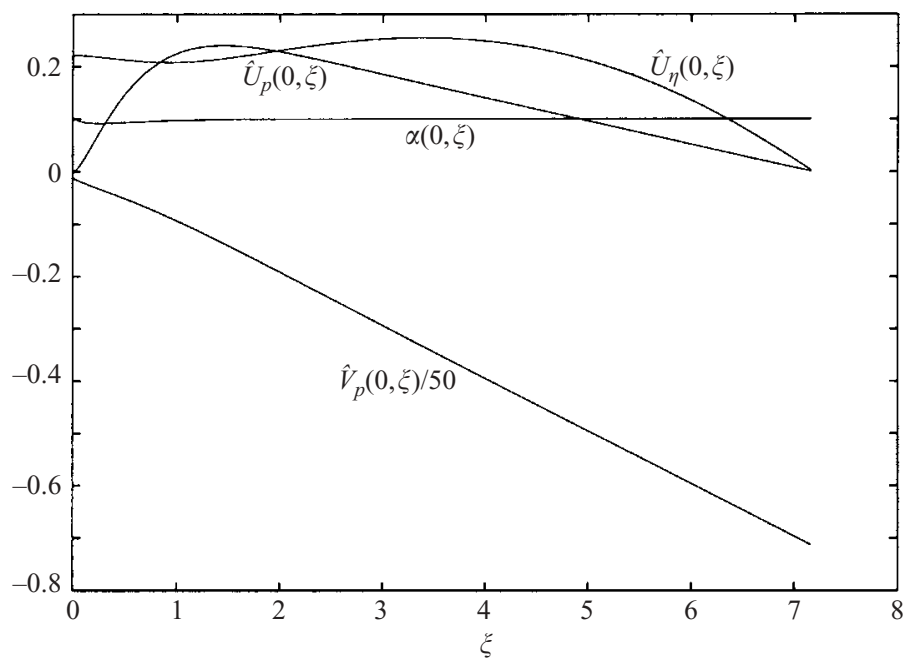

FIGURE 4. Downstream development of surface quantities, $\mathscr{K}^{\prime}=5, \alpha_{\infty} \gamma=0.1$.

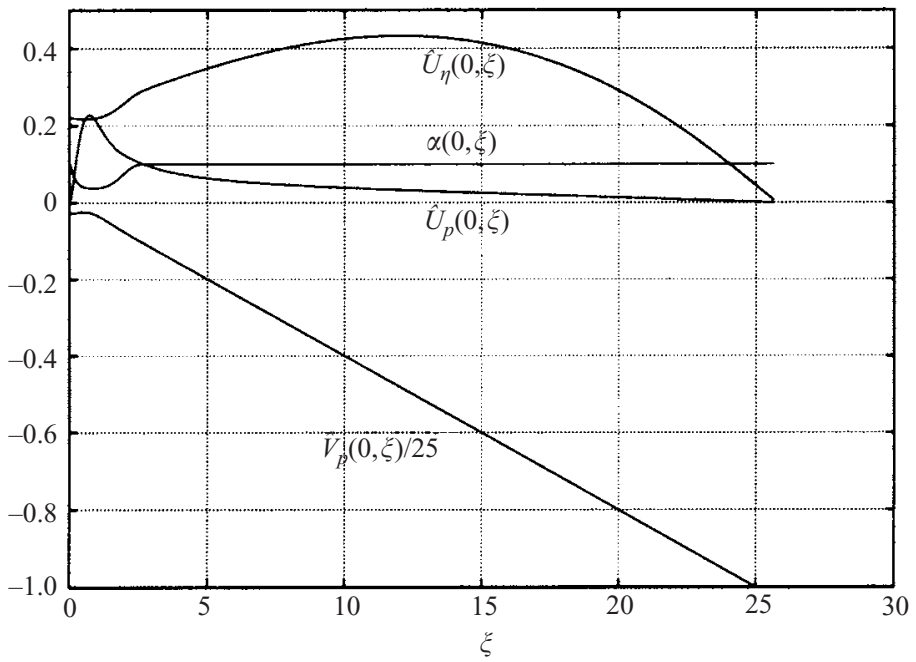

FIGURE 5. Downstream development of surface quantities, $\mathscr{K}^{\prime}=1, \alpha_{\infty} \gamma=0.1$.

development of surface fluid and particle quantities for the case $\mathscr{K}^{\prime}=5, \alpha_{\infty} \gamma=0.1$. What is observed from these distributions is the apparent simultaneous vanishing of the streamwise component of the particle velocity $\hat{U}_{p}(0, \xi)$ and the fluid wall shear stress $\hat{U}_{\eta}(0, \xi)$ at a critical downstream location $\xi \approx 7.16$. A similar calculation is shown for the case $\mathscr{K}^{\prime}=1$ (all other parameters unchanged) in figure 5 and this replicates the qualitative features of figure 4 . The computations confirm that in this case,

$$
\xi_{s}=\xi_{s}\left(\alpha_{\infty} \gamma, \mathscr{K}^{\prime}\right) .
$$

Again, the value of $\beta$ serves only to fix the location in $x^{*}$. 

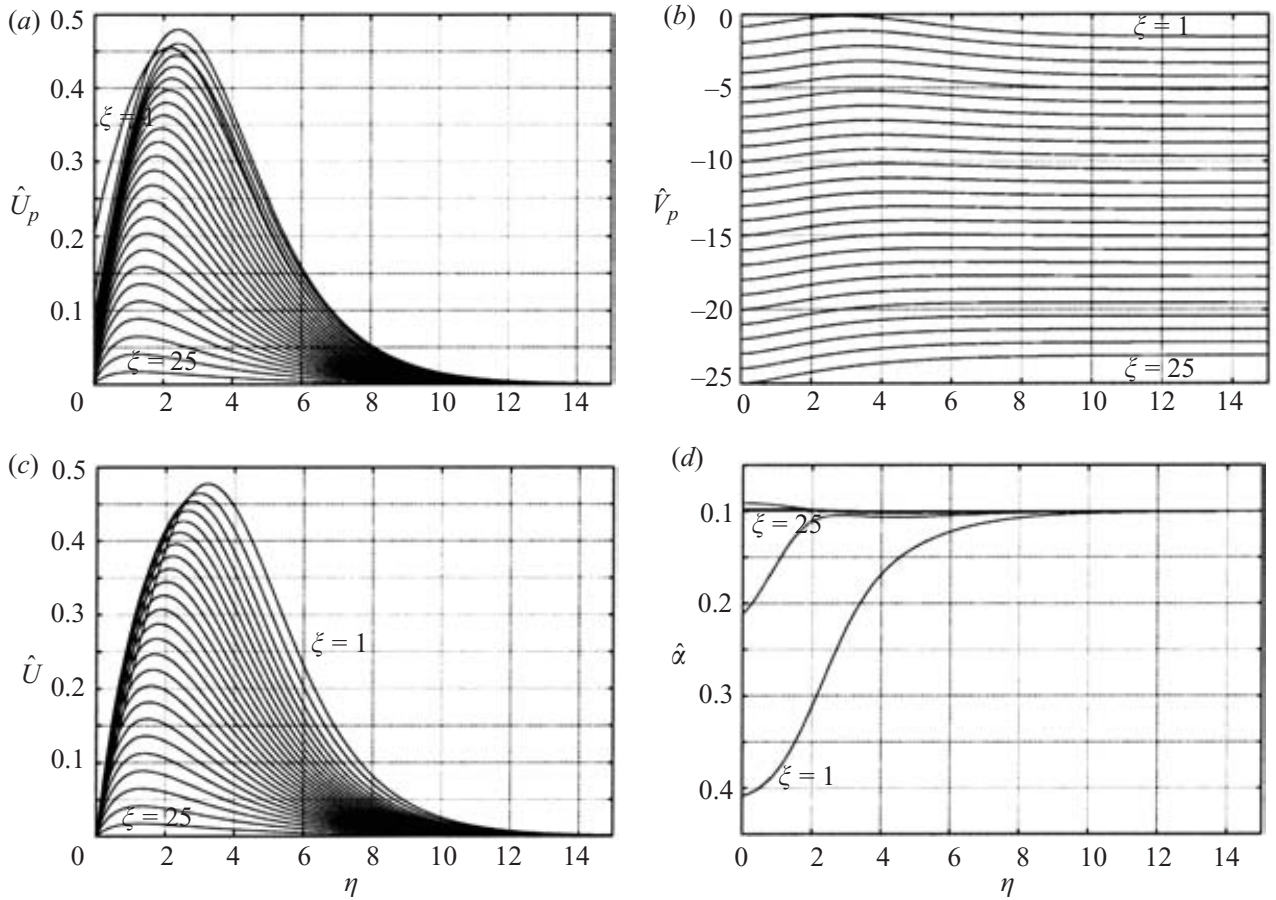

FIGURE 6. Flow and particle profiles, $\mathscr{K}^{\prime}=1, \alpha_{\infty} \gamma=0.1$.

Figures $6(a)-6(d)$ show the corresponding profiles for the flow and particles, and intriguingly indicate that the streamwise fluid velocity, $\hat{U}$ and the streamwise particle velocity $\hat{U}_{p}$ are both becoming identically zero across the entire jet at the same critical downstream location (in this case at $\xi=\xi_{s} \approx 25.6$ ). This is in contrast with the results of the preceding section in which both $\hat{U}_{p}$ and $\hat{V}_{p}$ vanished (just on the wall). Thus, in the absence of gravitational forcing, a stagnation point develops in the particle velocity field on the wall, whereas here the results point to a simultaneous flow reversal of both the particle and fluid velocity fields.

In the many computations performed by the authors to substantiate the results given above, a further point became apparent, namely that the approach to zero of $\hat{U}$ and $\hat{U}_{p}$ appears to be entirely regular, suggesting the following solution development as the critical downstream location, $\xi_{s}$ is approached:

$$
\begin{aligned}
\hat{U} & =\frac{4}{3} \xi_{s}\left(\xi_{s}-\xi\right) U^{*}(\bar{\eta})+\cdots, \\
\hat{V} & =\frac{4}{3} \xi_{s} V^{*}(\bar{\eta})+\cdots, \\
\hat{U}_{p} & =\frac{4}{3} \xi_{s}^{-1}\left(\xi_{s}-\xi\right) U_{p}^{*}(\bar{\eta})+\cdots, \\
\hat{V}_{p} & =\xi_{s} V_{p}^{*}(\bar{\eta})+\ldots, \\
\alpha & =\alpha^{*}(\bar{\eta})+\cdots,
\end{aligned}
$$

with $\bar{\eta}=\xi_{s} \eta$. Substitution into $(2.13 a)-(2.13 e)$ leads to the system

$$
\begin{gathered}
-U^{*}+V_{\bar{\eta}}^{*}=0, \\
-U^{* 2}+V^{*} U_{\bar{\eta}}^{*}=U_{\bar{\eta} \bar{\eta}}^{*}-\alpha^{*} \gamma\left(U^{*}-U_{p}^{*}\right),
\end{gathered}
$$




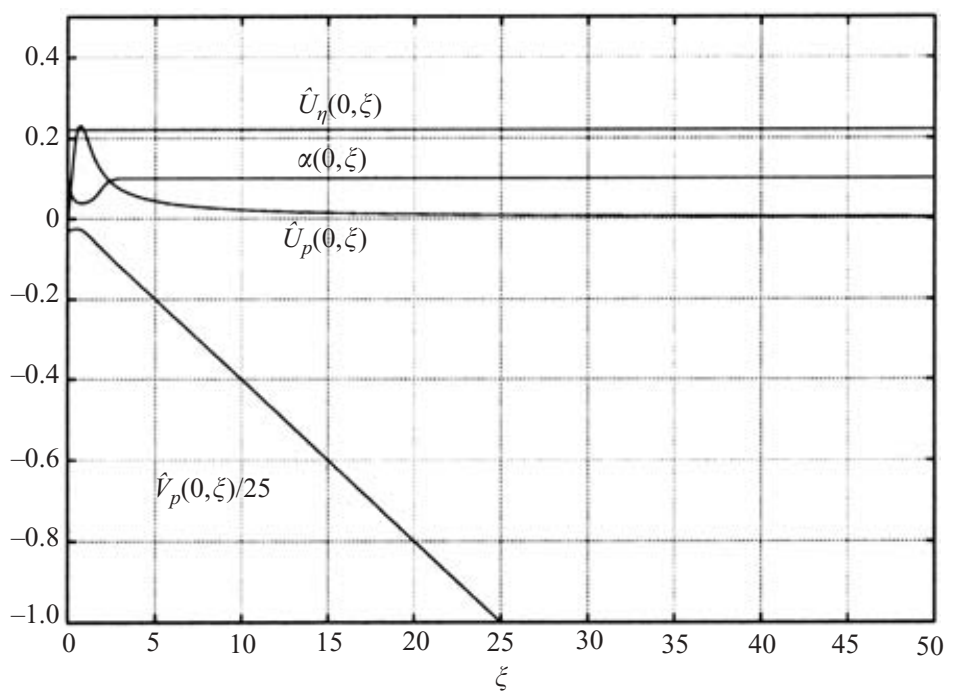

FIGURE 7. Downstream development of surface quantities in the dilute case with $\mathscr{K}^{\prime}=1$, $\alpha_{\infty} \gamma=0.1$.

$$
\begin{gathered}
-U_{p}^{* 2}+V_{p}^{*} U_{p \bar{\eta}}^{*}=U^{*}-U_{p}^{*}, \\
V_{p}^{*} V_{p \bar{\eta}}^{*}=V^{*}-V_{p}^{*}-\mathscr{K}^{\prime}, \\
V_{p}^{*} \alpha_{\bar{\eta}}^{*}+\alpha^{*} V_{p \bar{\eta}}^{*}-\alpha^{*} U_{p}^{*}=0 .
\end{gathered}
$$

That the flow stagnation seemingly occurs in a regular fashion is somewhat novel, given the singularities that are so often encountered in classical ('clean') boundarylayer flows around separation. Certainly, it is clear in this regime $\left(\mathscr{K}^{\prime}>0\right)$ that there is significant fluid/particle interaction. We note that the structure of this region is dependent on the values of two parameters: $\alpha_{\infty} \gamma$ and $\mathscr{K}^{\prime}$. We further note that this structure can be valid only for $\mathscr{K}$ of sufficient size to make $\mathscr{K}^{\prime}=O(1)$. Should this quantity be very small, then the structure of this zone reverts to that described in $\S 3$. Note also that the implication of the above from (2.15) is that

$$
\int_{0}^{\xi_{s}} \int_{0}^{\infty} \xi \hat{U} \int_{\eta}^{\infty} \gamma \hat{\alpha}\left(\hat{U}-\xi^{2} \hat{U}_{p}\right) \mathrm{d} \bar{\eta} \mathrm{d} \eta \mathrm{d} \xi=-1 .
$$

The dilute limit (i.e. $\alpha \rightarrow 0$ ) is also of some interest in this (co-gravitationally forced) regime. As noted already, this limit is retrieved easily by setting $\hat{\alpha} \equiv 0$ in $(2.13 b)$, and as noted earlier leads us to the conclusion that $(\hat{U}(\eta, \xi), \hat{V}(\eta, \xi))=\left(\hat{U}_{0}(\eta), \hat{V}_{0}(\eta)\right)$, and so the fluid velocity is simply the Glauert similarity solution as the jet evolves downstream.

Figure 7 shows some results for the particulate phase in the case $\mathscr{K}^{\prime}=1$ and $\alpha_{\infty} \gamma=0.1$; for ease of comparison with the previous results, the particle concentration normalization was taken to be as before, although in line with our comments above, the Stokes-drag term was omitted from the streamwise fluid momentum equation. As such, these results are the dilute analogy to those shown in figure 5. In this case, the computation proceeded downstream, with no sign of any breakdowns/singularities or any hint of the streamwise particle velocity becoming zero at any finite downstream location. More particularly, in this limit it is easy to see from $(2.13 a)-(2.13 e)$ that we 


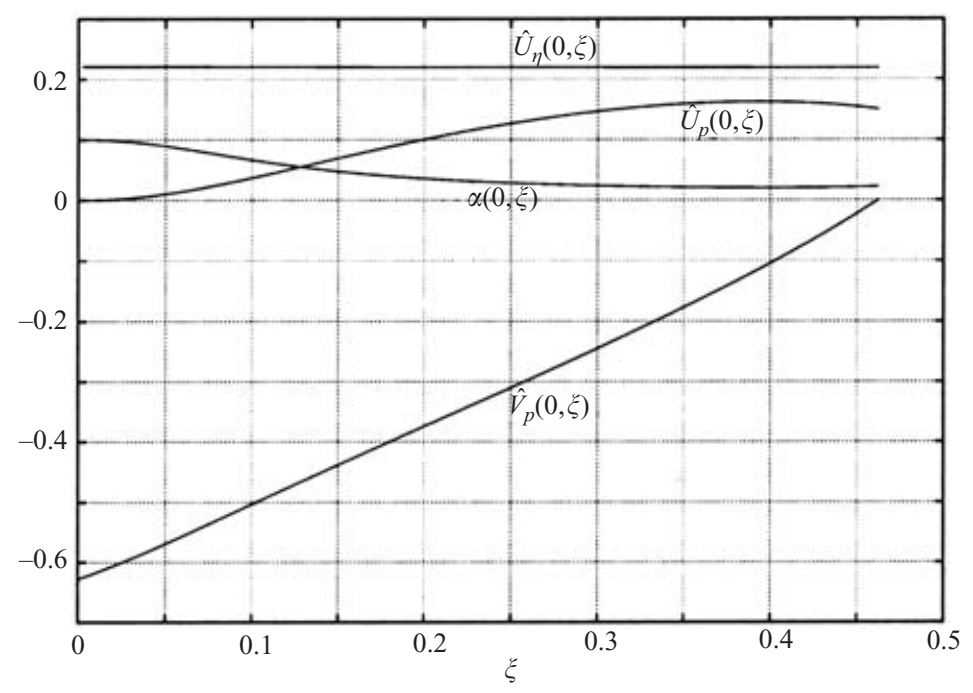

FIGURE 8. Downstream development of surface quantities, $\mathscr{K}^{\prime}=-1, \alpha_{\infty} \gamma=0.1$.

must have that

$$
\hat{U}_{p}(\eta, \xi) \rightarrow \hat{U}_{0}(\eta), \quad \hat{V}_{p}(\eta, \xi) \rightarrow-\mathscr{K}^{\prime} \xi+\hat{V}_{0}(\eta), \quad \hat{\alpha} \rightarrow \alpha_{\infty}
$$

and so far downstream the particle concentration becomes uniform (and equal to the free-stream value), the streamwise particle velocity becomes equal to the streamwise fluid velocity and the transverse particle velocity becomes equal to the gravitational 'settling' velocity (with a correction equal to that of the normal velocity of the fluid). In the non-dilute case, asymptotic inspection of $(2.13 b)$ and $(2.13 c)$ reveals this is not possible as $\xi \rightarrow \infty$, primarily because of inconsistencies caused by the presence of the Stokes-drag term in $(2.13 b)$. Thus, the mutual interaction between both phases is crucial in the development of the breakdown in this instance.

Results for the scenario where the particles are injected into the stream at some downstream location are given in the Appendix.

\section{The case $\mathscr{K}^{\prime}<0$ : flow under a boundary}

We have indicated already that when the direction of gravitational acceleration is counter to that of the transpiration induced by the jet, i.e. when the former acts in the direction away from the boundary, there exists the possibility of a balance existing between these two effects, leading to some interesting particle dynamics (cf. FDH).

In figure 8, we present results for the case $\mathscr{K}^{\prime}=-1$ and $\alpha_{\infty} \gamma=0.1$ (results which turn out to be representative for this regime). A critical streamwise position also exists in this case; here it is at $\xi \approx 0.46$, at which point the normal particle velocity at the wall becomes zero $\left(\hat{V}_{p}(\eta=0)=0\right)$. Calculations on a variety of grids led to entirely repeatable results up to this point; thereafter, although in most cases the calculations would continue for varying distances downstream, results were very sensitive to choices of the numerical grid. On the other hand, the streamwise wall shear stress of the fluid phase $\left(\hat{U}_{\eta}(\eta=0)\right)$ is seen to vary little downstream. It is of some interest to inspect the flow and particle profiles at $\xi=\xi_{s}$, and a selection of these are shown in figure 9. At this location, there is no indication of any singular behaviour in any of the profiles shown. The implications of these results are examined next, first close to 


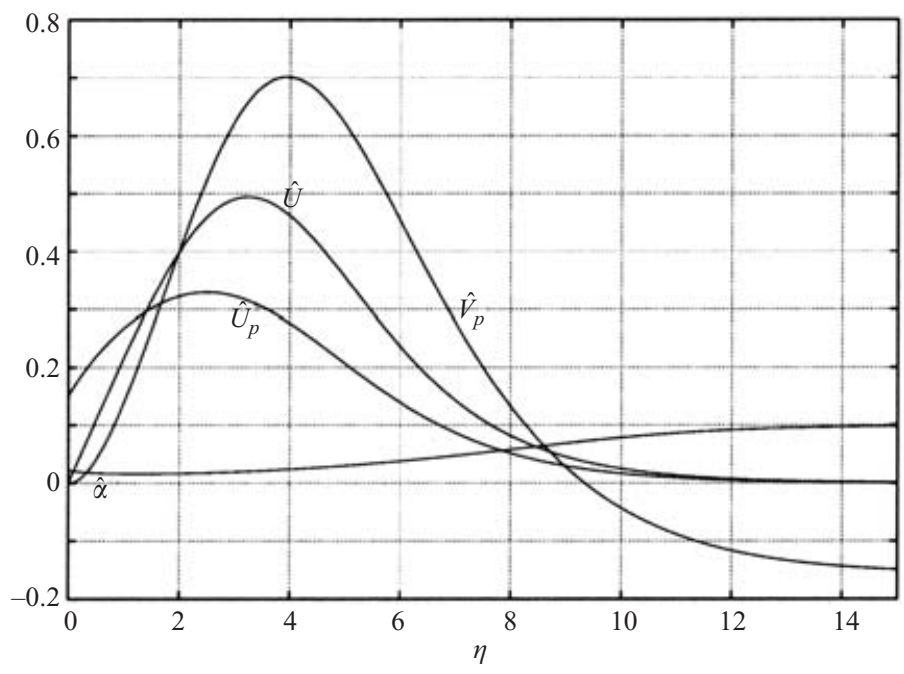

FIGURE 9. Solution profiles at breakdown $\left(\xi=\xi_{s}\right), \mathscr{K}^{\prime}=-1, \gamma \alpha_{\infty}=0.1$.

the location $\xi=\xi_{s}$, which gives some clues regarding the development of the solution further downstream.

\subsection{The solution around $\xi=\xi_{\text {s }}$}

There seems little doubt that the subtleties related to the computation of this flow are linked to the vanishing of $\hat{V}_{p}$ at the wall, all other flow quantities behaving in a regular fashion close to $\xi=\xi_{s}$. A number of corresponding dilute computations (i.e. with the decoupling of the particle velocities from the fluid momentum equations) were also performed, and yielded quantitatively similar results, reinforcing this view.

We now seek a local description of the flow just downstream of $\xi=\xi_{s}$ by first assuming that $\hat{V}_{p}(\eta=0)$ varies linearly around $\xi=\xi_{s}$, as strongly indicated in our numerical results. A balancing of terms in the $\hat{V}_{p}$ equation leads us to conclude that we should pursue a solution of the form

$$
\hat{V}_{p}=\frac{\xi-\xi_{s}}{\xi_{s}^{3}} V_{p}^{*}\left(\eta^{*}\right)+\cdots, \quad \hat{\alpha}=\alpha^{*}\left(\eta^{*}\right)+\cdots,
$$

where $\eta^{*}=\xi_{s}^{6} \eta /\left(\xi-\xi_{s}\right)^{2}$. Now supposing in the zone $\eta^{*}=O(1)$, as $\xi \rightarrow \xi_{s}$ that $\hat{U}_{p}=\hat{U}_{p 0}+\cdots$, where $\hat{U}_{p 0}$ is a constant, then we conclude that to leading order, $(2.13 d)$ becomes

$$
V_{p \eta^{*}}^{*}\left(V_{p}^{*}-\frac{3}{2} \eta^{*} \hat{U}_{p 0}\right)+\frac{3}{4} \hat{U}_{p 0} V_{p}^{*}=-\mathscr{K}^{\prime}
$$

Equation (5.2) indicates the likelihood of difficulties if $V_{p}^{*}=3 \eta^{*} \hat{U}_{p 0} / 2$, which will occur (only) if $\xi>\xi_{s}$. In order to match with the flow above the $\eta^{*}=O(1)$ zone (and by implication the flow ahead of $\xi=\xi_{s}$ ), it is necessary to impose the condition

$$
V_{p}^{*} \rightarrow-\frac{4 \mathscr{K}^{\prime}}{3 \hat{U}_{p 0}} \quad \text { as } \quad \eta^{*} \rightarrow \infty
$$


indeed, this solution identically satisfies (5.2). Helpfully, (5.2) can also be integrated to yield

$$
K_{1}=\frac{\left(V_{p}^{*}+\frac{4 \mathscr{K}^{\prime}}{3 \hat{U}_{p 0}}\right)^{2}}{V_{p}^{*}-\frac{3}{4} \hat{U}_{p 0} \eta^{*}+\frac{2 \mathscr{K}^{\prime}}{3 \hat{U}_{p 0}}},
$$

where $K_{1}$ is a constant of integration.

Consequently,

$$
V_{p}^{*}=\frac{1}{2}\left\{\left(K_{1}-\frac{8 \mathscr{K}^{\prime}}{3 \hat{U}_{p 0}}\right) \pm\left[\left(K_{1}-\frac{8 \mathscr{K}^{\prime}}{3 \hat{U}_{p 0}}\right)^{2}-4\left(\frac{16 \mathscr{K}^{\prime 2}}{9 \hat{U}_{p 0}^{2}}+\frac{3}{4} K_{1} \hat{U}_{p 0} \eta^{*}-\frac{2 \mathscr{K}^{\prime} K_{1}}{3 \hat{U}_{p 0}}\right)\right]^{1 / 2}\right\}
$$

Equation (5.3) corresponds to the choice $K_{1}=0$.

Sequentially, the particle volume fraction term may be determined (from the limiting form of (2.13e)), namely

$$
\left(V_{p}^{*}-\frac{3}{2} \hat{U}_{p 0} \eta^{*}\right) \alpha_{\eta^{*}}^{*}+\alpha^{*} V_{p \eta^{*}}^{*}=0,
$$

which may be routinely integrated to yield the general solution

$$
\alpha^{*}=K_{2} \exp \left\{-\int \frac{V_{p \eta^{*}}^{*}}{V_{p}^{*}-\frac{3}{2} \hat{U}_{p 0} \eta^{*}} \mathrm{~d} \eta^{*}\right\},
$$

where $K_{2}$ is a further constant of integration. It is not obviously apparent which are the correct/appropriate choices for the constants of integration $K_{1}$ and $K_{2}$. In order to obtain some guidance on this important detail, it is appropriate to consider a model version of the system (5.2) and (5.6), in which artificial viscosities are introduced into the system; although this technique is well known to be heuristic in many respects, nonetheless it can (and does here) yield valuable information regarding the system of interest here. Specifically, we modify (5.2) and (5.6) as follows:

$$
\begin{gathered}
V_{p \eta^{*}}^{*}\left(V_{p}^{*}-2 \eta^{*}\right)+V_{p}^{*}=-\hat{\mathscr{K}}^{\prime}+\tilde{\mu} V_{p \eta^{*} \eta^{*}}^{*}, \\
\left(V_{p}^{*}-2\right) \alpha_{\eta^{*}}^{*}+\alpha^{*} V_{p \eta^{*}}^{*}=\tilde{\mu} \alpha_{\eta^{*} \eta^{*}}^{*},
\end{gathered}
$$

(here, $V_{p}^{*}$ has been normalized with respect to $3 \hat{U}_{p 0} \xi_{s} / 4$ and $\hat{\mathscr{K}}^{\prime}$ denotes a suitably scaled $\mathscr{K}^{\prime}$ ). The quantity $\tilde{\mu}$ is the artificial viscosity, which we emphasize is aphysical, but nonetheless does give us useful guidance with regard to the nature of the solution to $(5.2)$ and (5.6) as $\tilde{\mu} \rightarrow 0$.

The inclusion of the two artificial viscosity terms clearly necessitates the need for the prescription of a value of both $V_{p}^{*}$ and $\alpha^{*}$ at the wall. The choice of these, thus far, has not been appropriate, but rather these wall values have themselves been part of the solution. With the inclusion of viscous terms, these choices are entirely arbitrary within our model (although both must be greater than or equal to zero). The exact microscale phenomenology of the particle-wall interaction may be regarded as being outside the (preliminary) scope of the 'dusty-gas' continuum model, and will only be elucidated by careful experimentation and comparison with the predictions derived 


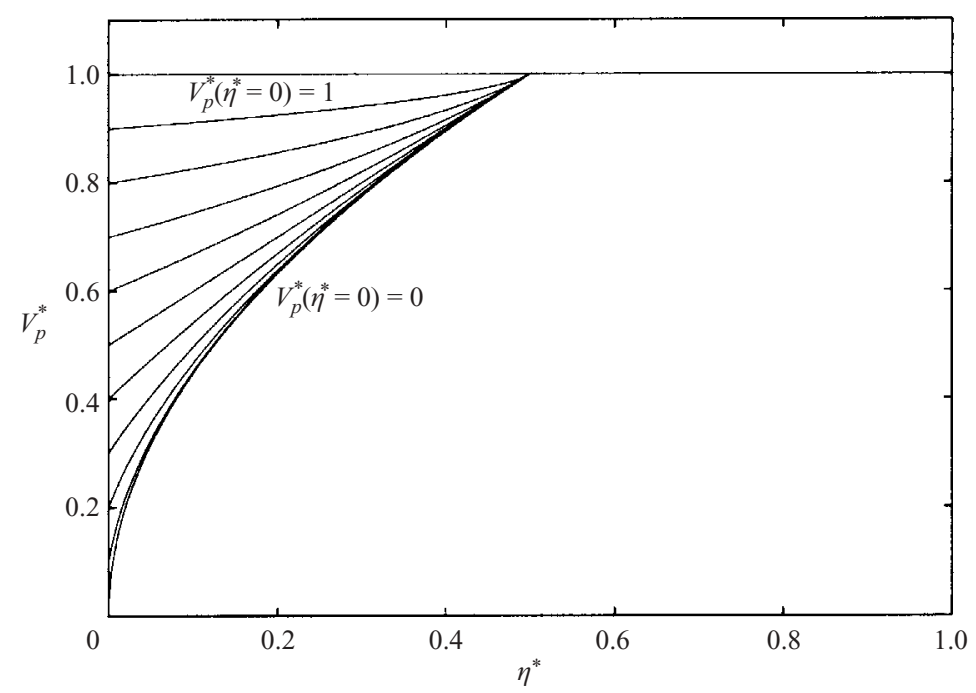

FIGURE 10. Distribution of $V_{p}^{*}$, for $\hat{\mathscr{K}}^{\prime}=-1, \tilde{\mu}=10^{-6}$ for $V_{p}^{*}(0)=0,0.1,0.2, \ldots, 1$.

both here and elsewhere. However, here we are merely introducing the small artificial viscosity and arbitrary wall conditions as a numerical mechanism to study the range of likely non-smooth solutions to the dusty-gas model in the limit of vanishing particle viscosity, our conjecture being that the global flow features are essentially independent of the particular ad hoc wall conditions that we choose to apply, as we shall verify below.

Equation (5.8) is then solved as a two-point boundary-value problem, using second-order finite differences, coupled with Newton iteration. Other parameter choices were that $\hat{\mathscr{K}}^{\prime}=-1$ and $\tilde{\mu}=10^{-6}$. Figure 10 shows distributions of $V_{p}^{*}$ for selected imposed values of $V_{p}^{*}\left(\eta^{*}=0\right)$, together with $V_{p}^{*} \rightarrow-\hat{\mathscr{K}}^{\prime}$ as $\eta^{*} \rightarrow \infty$. In spite of the smallness of $\tilde{\mu}$, some finite value was necessary for the success for these calculations, but equally the results shown in figure 10 may be regarded as converged with respect to the smallness of $\tilde{\mu}$. The striking feature of all the distributions shown is that beyond $\eta^{*}=0.5$, the value of $V_{p}^{*}$ is constant (and takes on the outer-limit value, $\left.-\hat{\mathscr{K}}^{\prime}\right)$; this value is attained at a (universal) value of $\eta^{*}=0.5$. The generic picture is therefore that the solution (for $V_{p}^{*}$ ) is dependent upon $V_{p}^{*}\left(\eta^{*}=0\right)$ for $0 \leqslant \eta^{*}<-\hat{\mathscr{K}}^{\prime} / 2$; beyond $\eta^{*}=-\hat{\mathscr{K}}^{\prime} / 2, V_{p}^{*}=-\hat{\mathscr{K}}^{\prime}$. It is especially gratifying to note that the solution above $\eta^{*}=-\hat{\mathscr{K}}^{\prime} / 2$ is entirely independent of our (arbitrary) choice of wall boundary condition.

A further feature of interest is that there are three choices of $V_{p}^{*}\left(\eta^{*}=0\right)$, which lead to especially simple solutions for $V_{p}^{*}$ for $0 \leqslant \eta^{*}<-\hat{\mathscr{K}}^{\prime} / 2$. The first of these, $V_{p}^{*}\left(\eta^{*}=0\right)=-\hat{\mathscr{K}}^{\prime}$ leads merely to $V_{p}^{*} \equiv-\hat{\mathscr{K}}^{\prime}$ (this is illustrated by the choice $V_{p}^{*}\left(\eta^{*}=0\right)=1$ in figure 10). If $V_{p}^{*}\left(\eta^{*}=0\right)=0$, then it is straightforward to show that $V_{p}^{*}=\left(-2 \hat{\mathscr{K}}^{\prime} \eta^{*}\right)^{1 / 2}$ for $0 \leqslant \eta^{*}<-\hat{\mathscr{K}}^{\prime} / 2$. The final (special) case is when $V_{p}^{*}\left(\eta^{*}=0\right)=-\hat{\mathscr{K}}^{\prime} / 2$, which permits a simple linear form, namely $V_{p}^{*}=-\hat{\mathscr{K}}^{\prime} / 2+\eta^{*}$.

Now that we have a clear picture of the behaviour of the normal particle velocity $V_{p}^{*}$, let us turn our attention to the particle distribution, $\alpha^{*}$, which may subsequently be determined. Consider first cases for which $V_{p}^{*}\left(\eta^{*}\right)=0$, which leads to the simple $V_{p}^{*}$ form as discussed above. The two-point boundary-value problem was then solved using second-order finite differences. Figure 11 shows the corresponding 


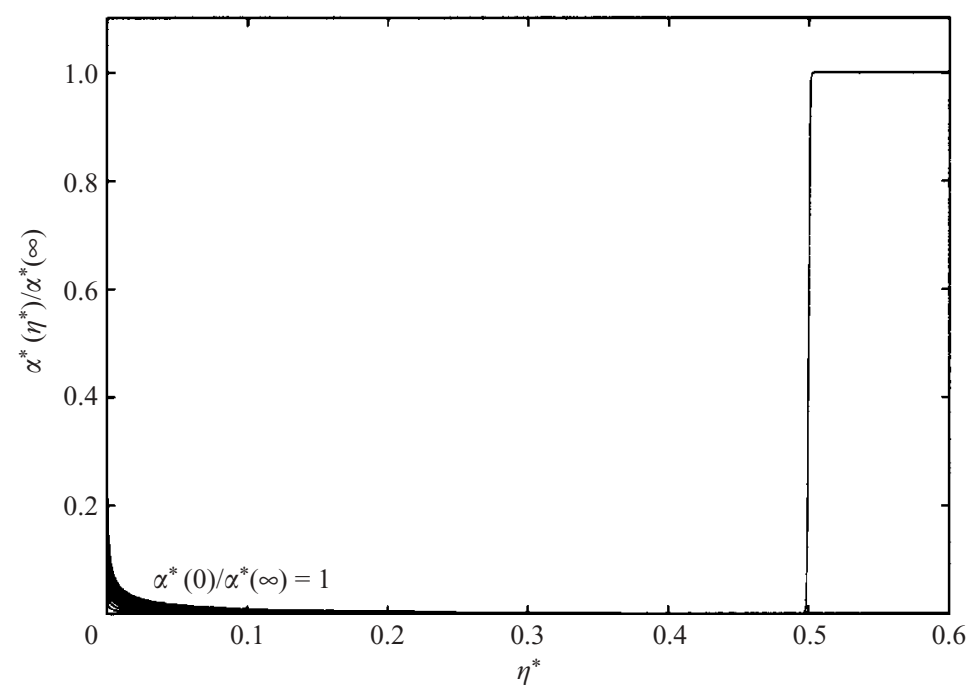

FIGURE 11. Distribution of $\alpha^{*}$, for $\hat{\mathscr{K}}^{\prime}=-1, V_{p}^{*}\left(\eta^{*}=0, \tilde{\mu}=10^{-6}\right.$ for $\alpha^{*}(0) / \alpha^{*}(\infty)=0,0.1,0.2, \ldots, 1$.

distributions for $\alpha^{*}\left(\eta^{*}\right) / \alpha^{*}(\infty)$ for selected imposed wall values. These results point to the striking result that below the critical location $\eta^{*}=-\hat{\mathscr{K}}^{\prime} / 2, \alpha^{*}$ shows a strong tendency to take on a value of zero; at this critical point, all the indications are of the formation of a shock, at which point the distribution jumps to the upper $\left(\eta^{*} \rightarrow \infty\right)$ value. This important observation points to the conclusion that $\alpha^{*}(\eta=0)=0$ is the appropriate choice, and indeed this value is maintained up to the critical (shock) location $\eta^{*}=-\hat{\mathscr{K}}^{\prime} / 2$, indicating the flow to be particle-free below the shock. Decreasing the artificial viscosity led to confirmation of this observation. (Indeed an analogous phenomenon was found by FDH.) Taking the particular choice $\alpha^{*}\left(\eta^{*}=0\right)=0$, the authors computed the $\alpha^{*}$ distributions corresponding to the $V_{p}^{*}$ distributions shown in figure 10 and these confirmed the conclusion that $\alpha^{*}=0$ for $0 \leqslant \eta^{*}<-\hat{\mathscr{K}}^{\prime} / 2, \alpha^{*} / \alpha^{*}\left(\eta^{*}=\infty\right)=1$ for $\eta^{*}>-\hat{\mathscr{K}}^{\prime} / 2$. Finally, it is worth pointing out that the fluid velocity is little affected by all the subtleties encountered in the particle phase.

\subsection{The solution beyond $\xi=\xi_{\text {s }}$}

Since the concept of the inclusion of artificial viscosity has proved to be a useful technique in the localized problem around $\xi=\xi_{s}$, we now move on to incorporate this into our treatment of the full system. Specifically, this was achieved by adding in additional 'viscous' terms into the governing system.

The inclusion of these additional second-order terms obviously necessitates the prescription of additional boundary conditions (on $\eta=0$ ), which are entirely arbitrary. A sensible choice to model these, however, appears to be to choose values downstream of the critical location $\xi_{s}$ to take on the values found at $\xi=\xi_{s}$, i.e.

$$
\hat{V}_{p}\left(\eta=0, \xi>\xi_{s}\right)=0, \quad \text { i.e. } \quad \tilde{V}_{p}\left(\eta=0, \xi>\xi_{s}\right)=\frac{\gamma \mathscr{K}^{\prime} \xi}{\beta}-\hat{V}_{0}(\infty)
$$


(a)

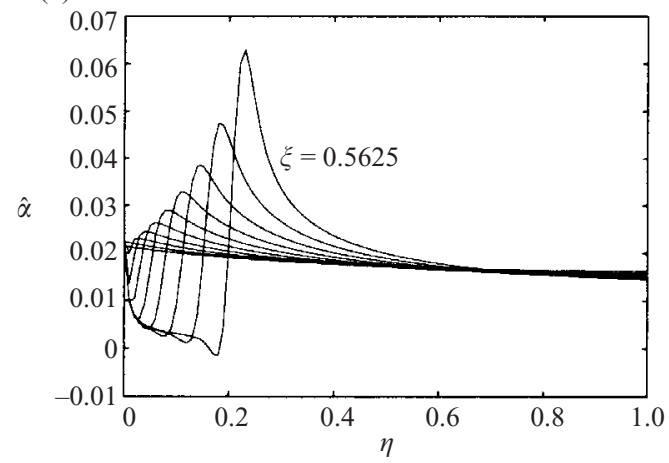

(b)

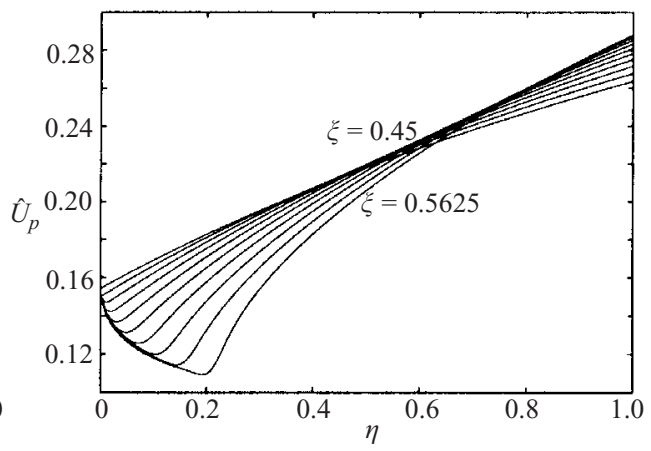

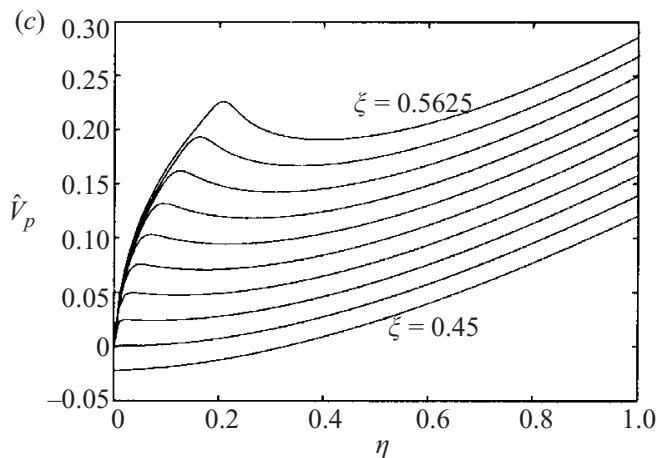

FIGURE 12. Near-wall particle distribution and velocity profiles, $\mathscr{K}^{\prime}=-1, \gamma \alpha_{\infty}=0.1$, $\mu^{*}=10^{-8}$.

$\hat{U}_{p}\left(\eta=0, \xi>\xi_{s}\right)=\hat{U}_{p}\left(\eta=0, \xi=\xi_{s}\right), \quad$ i.e. $\quad \tilde{U}_{p}\left(\eta=0, \xi>\xi_{s}\right)=\frac{\xi_{s}^{2}}{\xi^{2}} \tilde{U}_{p}\left(\eta=0, \xi=\xi_{s}\right)$

$\hat{\alpha}\left(\eta=0, \xi>\xi_{s}\right)=\hat{\alpha}\left(\eta=0, \xi=\xi_{s}\right), \quad$ i.e. $\quad \tilde{\alpha}\left(\eta=0, \xi>\xi_{s}\right)=\frac{\xi_{s}^{2}}{\xi^{2}} \tilde{\alpha}\left(\eta=0, \xi=\xi_{s}\right)$.

Solution profiles for the particle phase for $\beta=\gamma=1, \mathscr{K}^{\prime}=-1$ (cf. figure 8) are shown in figures $12(a)-12(c)$ at downstream locations $\xi=0.45$ (immediately upstream of $\xi_{s}$ in this case), $0.4625,0.475, \ldots, 0.5625$. For these calculations, the value of the artificial viscosity parameter $\mu^{*}$ was taken to be $10^{-8}$; results are presented close to the wall, close to the location of the shock. From the $\hat{\alpha}$ distributions (figure 12a) there is clear evidence of the formation of a shock progressively downstream, and these results (crucially) reinforce the view that the region below the shock is becoming particle-free. The computation was suspended downstream of $\xi=0.5625$ to avoid aphysical negative values of $\hat{\alpha}$. The $\hat{U}_{p}$ distributions (figure $12 b$ ) indicate that this quantity remains continuous across the shock, a feature replicated in the $\hat{V}_{p}$ distributions (figure 12c). Just beyond the final downstream location presented, the calculations ceased to converge. One likely cause of this is that it is inevitable that in the outer edges of the jet the direction of $\hat{V}_{p}$ will also ultimately change, a detail highlighted by figure 13, which again shows the $\hat{V}_{p}$ distributions of figure $12(c)$, but across the entire jet profile. Indeed, since $\hat{V}_{0}(\infty) \approx-0.628$, for this particular choice of parameters the dilute-limit problem will ultimately encounter this difficulty at $\xi \approx 0.628$. This (further) change in direction in $\hat{V}_{p}$ at the outer edge of the jet 


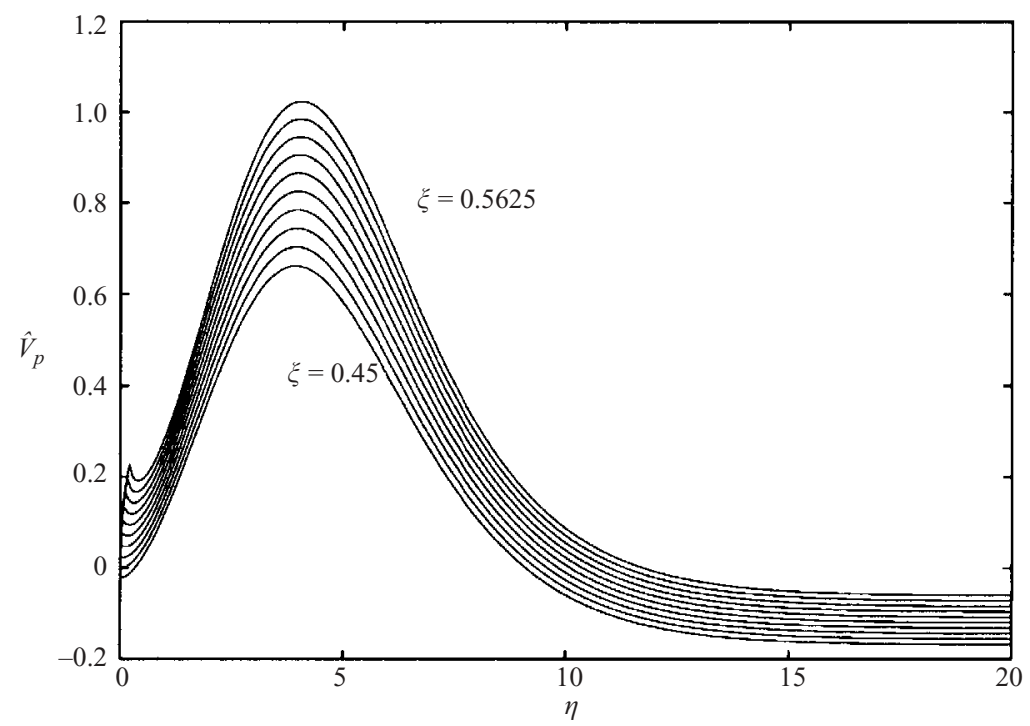

FIGURE 13. Distribution of $\hat{V}_{p}$ across jet (parameters as for figure 12c).

(in addition to the earlier change at the wall) presents a significant difficulty in extending these computations further downstream, and it was decided to halt the computations prior to this point. Finally, it should be noted that the fluid (again) behaves in a relatively benign fashion in this region.

\section{Effects of the Saffman force}

The singularity that occurs at $\xi=\xi_{s}$, at least for the $\mathscr{K}^{\prime}=0$ case, calls into question the validity of this 'dusty-gas' model in the vicinity of the singularity since the volume fraction takes unphysically large values. The natural question that arises is how this singularity is to be resolved in the broader context of more general field equations. Possibilities include extensions that incorporate finite particle volume-fraction effects, or the (neglected) Saffman force, in order to resolve the singularity within the confines of the dilute-limit equations.

Some authors have found that inclusion of the Saffman force acts to remove these unphysical singularities from the particle fluid. Osiptsov (1988) found that the singularity at the wall in the Blasius flow is strongly modified by the inclusion of the Saffman force. We have given the appropriate form for the force in this problem in (2.19); so we can now assess its influence in the singularity structure.

In the $\mathscr{K}^{\prime}=0$ case of $\S 3,(3.2 b)$ is modified by the presence of the Saffman force to be

$$
-\hat{V}_{0} \hat{U}_{p 0}+\hat{\eta} \hat{U}_{p 0} \hat{V}_{p 0 \hat{\eta}}+\hat{V}_{0} \hat{V}_{p 0 \hat{\eta}}=-\hat{V}_{p 0}+\epsilon\left[\hat{U}_{\eta}\left(\eta=0, \xi_{s}\right) \hat{\eta}-\hat{U}_{p 0}\right]
$$

where the multiplier of the new term is given by

$$
\epsilon=c\left(\frac{2}{9}\right)^{3 / 4} \frac{R e_{p}^{3 / 2}}{\gamma^{1 / 4} \xi_{s}^{1 / 2}}\left|\hat{U}_{\hat{\eta}}\left(\hat{\eta}=0, \xi_{s}\right)\right|^{1 / 2} .
$$

Thus, the Saffman force does indeed modify the equation for $\hat{V}_{p 0}$, but it does not alter the structure itself, since the local similarity is preserved, and therefore the stagnation point for $\hat{U}_{p}$, and most importantly the singularity in $\alpha$ remain. 
In $\S 4$, the equations that describe the flow near the singularity are not modified at all by the Saffman force. The reason is that it vanishes at the wall, but the gravitational force does not. Hence, there is no effect to leading order.

Finally, in $\S 5$, the equation obtained for $V_{p}^{*}$ is modified by the Saffman force, so that (5.2) becomes

$$
\begin{gathered}
V_{p \eta^{*}}^{*}\left(V_{p}^{*}-\frac{3}{2} \eta^{*} \hat{U}_{p 0}\right)+\frac{3}{4} \hat{U}_{p 0} V_{p}^{*}=-\mathscr{K}^{\prime}-\epsilon^{\prime} \hat{U}_{p 0}, \\
\epsilon^{\prime}=c\left(\frac{2}{9}\right)^{3 / 4} \gamma^{-1 / 4} \operatorname{Re}_{p}^{3 / 2} \xi_{s}^{7 / 2}\left|\hat{U}_{\eta}\left(\eta=0, \xi_{s}\right)\right|^{1 / 2} .
\end{gathered}
$$

In this case, the only effect seems to be to modify the apparent value of $\mathscr{K}^{\prime}$, making $\left|\mathscr{K}^{\prime}\right|$ smaller than it would have been with no Saffman force.

The Saffman force is clearly very small for all order-one $\xi$ since $R e_{p}^{3 / 2} \ll 1$. Since the term is entirely regular, it can have no effect over the range of the numerical integration. However, because it depends on the shear of the fluid velocity, there is always a possibility that even at asymptotically small $R e_{p}$ the term might become important near the singularity, thus modifying the structure. Nonetheless, the above analysis indicates that the near-singularity structure is preserved in all three cases, and that for $\mathscr{K} \leqslant 0$, a small modification in the solutions in those regions arises, but in both cases the alterations do not affect the structure of the solutions in those zones.

\section{Conclusions}

In this paper, we have described the spatial development of a wall-jet flow in a particle-laden incompressible viscous fluid. The modelling approach we have adopted is the 'dusty-gas' equations, which treat the flow as two continua that interact through a Stokes-drag mechanism. In the absence of any particle suspension, there is a wellknown similarity solution to this problem due to Glauert (1956). However, there is no such self-similar solution available in the fully coupled particle-fluid interaction problem.

There are three distinct cases described herein: (i) insignificant gravitational forcing $\left(\left|\mathscr{K}^{\prime}\right| \ll 1\right)$; (ii) a jet flow above a boundary $\left(\mathscr{K}^{\prime}>0\right)$; and (iii) a jet flow below a boundary $\left(\mathscr{K}^{\prime}<0\right)$. The distinction between $\mathscr{K}^{\prime}>0$ and $\mathscr{K}^{\prime}<0$ is that in the former, gravitational effects act in conjunction with the fluid entrainment into the jet, whereas in the latter case these two mechanisms compete. Having derived the governing equations, it is necessary to take great care to build in the correct nearorigin asymptotic behaviour and solve the resulting system via a spatial marching technique to determine the downstream evolution accurately.

In case (i), $\left|\mathscr{K}^{\prime}\right| \ll 1$, we have seen that the downstream evolution of the flow terminates with the generation of a stagnation point in the particle phase on the wall. This stagnation point leads to a singularity in the particle concentration violating the assumption of the model that the particles are non-colloidal. Therefore, beyond this critical location we must return to a more general set of field equations to determine the evolution of the flow.

In case (ii), $\mathscr{K}^{\prime}>0$, gravitational effects act in conjunction with the transverse flow induced by the jet to drive particles towards the bounding wall. In this case, the spatial development (in the context of the 'dusty-gas' model) again breaks down through the simultaneous reversal (across the entire jet) of both the fluid and particle flow at a critical downstream location. This evolution depends crucially on the interaction of the particle and fluid phases, being absent if the two systems of equations decouple. 
In case (iii), $\mathscr{K}^{\prime}<0$, gravitational effects act to force particles away from the boundary whilst the entrainment of fluid by the jet acts to force particles towards the boundary. Since the jet entrainment decays spatially as the flow evolves downstream, this also leads to some interesting behaviour. The physical picture in this case is more straightforward than the mathematical subtleties of $\S 5$ might suggest. A particlefree region near the wall develops at a critical downstream location, caused by the gravitational forcing of particles away from the boundary. This particle vacuum is separated from the outer particle-filled flow by a shock in the particle concentration distribution. Although we can solve 'below' the shock with arbitrary wall values imposed in the model, the influence of these arbitrary conditions appears to be felt neither in the fluid phase nor in the exterior particle-filled region of the flow and act only to adjust the velocity field within the vacuum region. This makes intuitive sense, since the governing 'dusty-gas' equations, when cast in their conservative form (see below), are entirely trivial in regions for which the particle concentration is zero, $\alpha=0$, and remain decoupled from the fluid phase. This analysis lends strong support to the geometrically more complex numerical engineering studies of particle-laden flows that allow boundary conditions to be imposed on a particle phase in flow regions where no such particles exist, effectively solving for 'virtual particles'. Summarizing, the breakdown is located at

$$
x_{s}^{*}=\frac{v}{M}\left(\frac{9 \xi_{s}^{2}}{9}\right)^{2 / 3} R e_{p}^{-4 / 9}, \quad \xi_{s}=F\left(\mathscr{K}^{\prime}, \alpha_{\infty} \gamma\right),
$$

where the function of the two parameters, $F$, has been computed for several cases, with qualitatively different behaviours near $\xi_{s}$ depending on the sign of $\mathscr{K}^{\prime}$.

Arguments based on characteristics are also useful in this context, and further reinforce the conclusions detailed above. In particular, the three 'particle' equations $(2.5 c)-(2.5 e)$ are hyperbolic, all with characteristics given by

$$
\frac{\mathrm{d} Y}{\mathrm{~d} x}=\frac{V_{p}}{U_{p}} .
$$

Ahead of the critical downstream location $\xi_{s}$, the particle velocity in the neighbourhood of the wall is clearly directed vertically downwards (see figure 8), the implication being (and confirmed by figure 12c) that beyond this point, the particle velocity becomes directed vertically upwards, which in turn leads to characteristics intersecting, with the possibility of shocks forming. It is possible (and straightforward) to write $(2.5 c)-(2.5 e)$ in conservation form, and the presumption that the zone below the shock is particle free, leads to the conclusion (from all of $(2.5 c)-(2.5 e)$ ) that the shock location is determined from

$$
\frac{\mathrm{d} Y_{s}}{\mathrm{~d} x}=\frac{V_{p}^{s}}{U_{p}^{s}}
$$

where $\left(U_{p}^{s}, V_{p}^{s}\right)$ is the particle velocity at the shock (it is our assertion that this is continuous). The results of our local analysis around $\xi=\xi_{s}$ are entirely consistent with these observations. One final point, in this respect, is that an analogous event happens further downstream, where there is a change in direction of the normal component of the particle velocity, $V_{p}$, at the outer edge of the jet, which in turn leads to the intersection of characteristics in that region also.

In each of the above cases, asymptotic descriptions are provided in the neighbourhood of the stagnation point, the flow reversal and the particle-free region, respectively. We have described a simple boundary-layer flow in a straightforward 


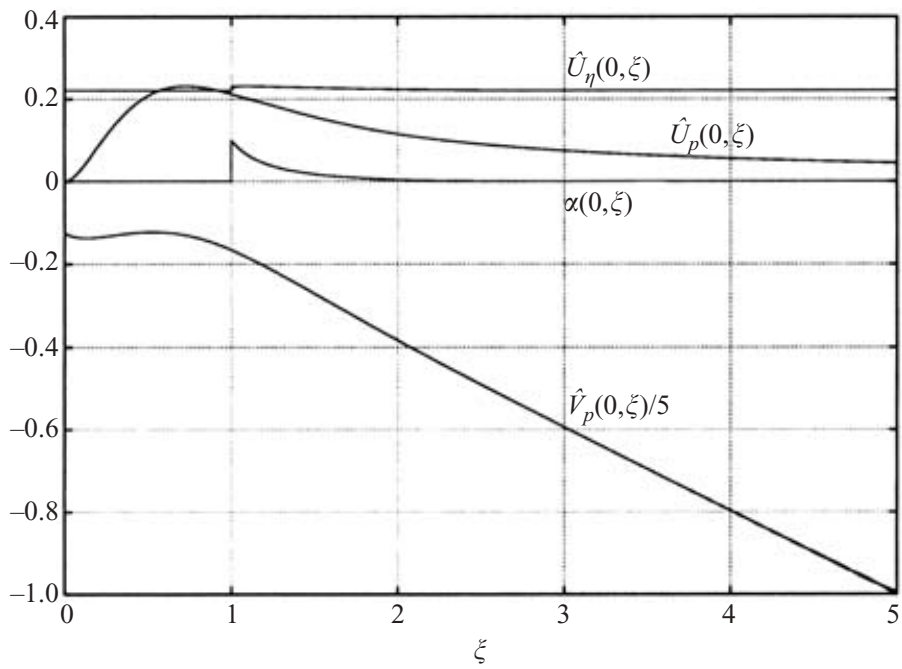

FIGURE 14. Downstream development of surface quantities, $\mathscr{K}^{\prime}=1$, $\alpha(\eta, \xi=1)=\alpha_{\text {profile }}(\eta)$.

and experimentally realizable configuration, making definite quantitative and qualitative predictions regarding the flow evolution. It is hoped that future careful experimentation will interact with the theoretical predictions described above to drive further development of the model equations and validation of the 'dusty-gas' approach over a broad parameter range.

The authors wish to express their gratitude to Professor Peter Davies for a number of useful discussions connected with the physical aspects of the problem. The support of the EPSRC is gratefully acknowledged. Some final work on the manuscript was accomplished while the authors were in residence at the Isaac Newton Institute for the Mathematical Sciences, whose support we also acknowledge. An anonymous referee provided a number of useful comments on an earlier version of this paper.

\section{Appendix. Injection of particles at $\xi=1$}

As an example of a case in which the particles are introduced into the jet at a finite downstream location (i.e. rather than being introduced into the jet $a b$ initio), we now consider a (full non-dilute) case for which we specify

$$
\hat{\alpha}(\eta, \xi=1)=\alpha_{\text {profile }}(\eta)=0.1 e^{-\eta},
$$

together with $\alpha_{\infty}=0, \beta=\gamma=1$. With regard to the treatment of $\hat{U}_{p}$ and $\hat{V}_{p}$, there are obviously many (arbitrary) choices here, but we chose to continuously compute these quantities from $\xi=0$, downstream, treating the location $\xi=1$ just like any other. Clearly, between $\xi=0$ and $\xi=1, \hat{U}_{p}$ and $\hat{V}_{p}$ may be regarded as 'virtual velocities', although they do represent the particle velocity components in the dilute limit. The flow development observed is quite different (but nonetheless physically reasonable) from the previous examples presented, as shown in figure 14. In particular, neither the flow nor particle motion show any signs of stagnation, and the calculation proceeded downstream, unabated, with a progressive dilution of the fluid particles, as would be very much expected. In particular, inspection of the governing 
equations $(2.13 a)-(2.13 e)$ in the far downstream limit indicates that

$$
\begin{gathered}
\hat{U}_{p}(\eta, \xi) \rightarrow \hat{U}(\eta, \xi) \rightarrow \hat{U}_{0}(\eta), \quad \hat{V}(\eta, \xi) \rightarrow \hat{V}_{0}(\eta), \\
\hat{V}_{p}(\eta, \xi) \rightarrow \hat{V}_{0}(\eta)-\mathscr{K}^{\prime} \xi, \quad \hat{\alpha} \rightarrow 0,
\end{gathered}
$$

and so far downstream the fluid reverts to the Glauert form, the particle distribution becomes progressively more dilute, with a streamwise velocity equalling that of the fluid, and a normal velocity equalling the buoyancy/settling velocity (with a correction term equal to the normal velocity of the fluid). There is therefore a clear, qualitative difference with the flow and particle behaviour in this case (when compared with the earlier corresponding nonlinear calculations shown in this section, which led to the formation of a stagnation point). The cause of this key difference appears to be the fluid/particle interaction term in $(2.13 b$ ) (in particular, if $\alpha$ takes a finite value at the outer edge of the jet), which prohibits an asymptotic form along the lines of (A $2 a)$.

\section{REFERENCES}

Asmolov, E. S. 1992 Motion of a dispersed admixture in a laminar boundary layer on a flat plate. Izv. Akad. Nauk SSSR Mekh Zhidk Gaza N1, 66.

FÉraille, T. \& Casalis, G. 2003 Channel flow induced by wall injection of fluid and particles. Phys. Fluids 15, 348.

Foster, M. R., Duck, P. W. \& Hewitt, R. E. 2003 The unsteady Kármán problem for a dilute suspension. J. Fluid Mech. 474, 379.

Glauert, M. B. 1956 The wall jet. J. Fluid Mech. 1, 625.

Gundogdu, O., Koenders, M. A., Wakeman, R. J. \& Wu, P. 2003 Vibration-assisted dead-end filtration. Experiments and theoretical concepts. Trans. Instn Chem. Engrs 81, 916.

Hernández, J. A. 2001 Instabilities induced by concentration gradients in dusty gases. J. Fluid Mech. 435, 247.

JACKSON, R. 1996 Locally averaged equations of motion for a mixture of identical spherical particles in a Newtonian fluid. Chem. Engng Sci. 52, 2457.

Jones, A. V. \& ProsperetTi, A. 1985 The suitability of first-order differential models for two-phase flow prediction. Intl J. Multiphase Flow 11, 133.

LeE, S. L. \& Chan, W. K. 1972 Two-phase laminar boundary layer along a vertical flat wall. Hydrotransport 2, 58.

LiU, J. T. C. 1966 Flow induced by an oscillating infinite flat plate in a dusty gas. Phys. Fluids $\mathbf{9}$, 1716.

McTigue, D. \& Jenkins, J. 1992 Channel flow of a concentrated suspension. Advances in Micromechanics of Granular Material (ed. H. Shen). Elsevier.

Marble, F. E. 1963 Dynamics of a gas containing small solid particles. Combustion and Propulsion, 5th AGARD Colloquium, Oxford. Pergamon.

Marble, F. E. 1970 Dynamics of dusty gases. Annu. Rev. Fluid Mech. 2, 397.

Michael, D. H. 1968 The steady motion of a sphere in a dusty gas. J. Fluid Mech. 31, 175.

Narayanan, C. \& Lakehal, D. 2002 Temporal instabilities of a mixing layer with uniform and nonuniform particle loadings. Phys. Fluids 14, 3775.

Osiptsov, A. N. 1980 Structure of a dispersed-mixture laminar boundary layer on a flat plate. Izv. Akad Nauk SSSR Mekh Zhidk Gaza N4, 48.

Osiptsov, A. N. 1988 Dusty gas motion in the initial section of a plane channel or circular pipe. Izv. Akad. Nauk SSSR Mekh Zhidk Gaza N6, 80.

Osiptsov, A. N. 1997 Mathematical modeling of dusty-gas boundary layers. Appl. Mech. Rev. 50, 357.

Prosperetti, A. \& Jones, A. V. 1985 The linear stability of general two-phase flow models-II. Intl J. Multiphase Flow 13, 161.

SAFFmAn, P. G. 1965 The lift of a small sphere in a slow shear flow. J. Fluid Mech. 22, 385. (See also Corrigendum, 1968, 31, 624.) 
Singleton, R. E. 1965 Compressible gas-solid particle flow over a semi-infinite flat plate. Z. Angew. Math. Phys. 16, 421.

Slater, A. S. \& Young, J. B. 2001 The calculation of inertial particle transport in dilute gas-particle flows. Intl J. Multiphase Flow 27, 61.

Soo, S. L. 1968 Non-equilibrium fluid dynamics - laminar flow over a flat plate. Z. Angew. Math. Phys. 19, 545.

Ungarish, M. \& Greenspan, H. P. 1983 On two-phase flow in a rotating boundary layer. Stud. Appl. Maths 69, 145.

WANG, B. Y. \& Glass, I. I. 1988 Compressible laminar boundary-layer flows of a dusty gas over a semi-infinite flat plate. J. Fluid Mech. 186, 223.

Zhang, D. Z. \& Prosperetti, A. 1997 Momentum and energy equations for disperse two-phase flows and their closure for dilute suspensions. Intl J. Multiphase Flow 23, 425. 